\title{
Two Simultaneously Occurring Potamogeton Species: Similarities and Differences in Seasonal Changes of Biomechanical Properties
}

\author{
Anna Maria Koboda ${ }^{1 *}$, Robert Józef Bialik ${ }^{2}$, Mikołaj Karpiński ${ }^{1}$, \\ Lukasz Przyborowski ${ }^{1}$ \\ ${ }^{1}$ Institute of Geophysics, Polish Academy of Sciences, Warsaw, Poland \\ ${ }^{2}$ Institute of Biochemistry and Biophysics, Polish Academy of Sciences, Warsaw, Poland
}

Received: 29 September 2017

Accepted: 10 February 2018

\begin{abstract}
Two common Potamogeton species, Potamogeton pectinatus L. and Potamogeton crispus L., were collected in 2016 and 2017 from a lowland, sandy bed in the Wilga River in Poland to investigate the ability of the plants to adapt to changing hydrological conditions. Measurements included biomechanical properties as well as the morphological characteristics of their stems. Specifically, experiments included three-point bending and tension tests as well as stem diameter and cross-sectional morphology at various periods in the plants' life cycles. Detailed information about the seasonal changes in biomechanical traits and the similarities between the two investigated plants are presented. The data show significant differences in the three-point bending and tension parameters. The flexural rigidity proved to be the most sensitive parameter to changes in hydrological conditions during the season. The maximum forces in the three-point bending tests needed to complete the fracture of $P$. crispus were much higher, reaching values up to $0.097 \mathrm{~N}$, than those for P. pectinatus $(0.035 \mathrm{~N})$, due to P. crispus having thicker shoots, which resulted in greater resistance to elastic deformity. Moreover, the modulus of elasticity values shows that $P$. pectinatus is much more prone to return to its original shape after the removal of the acting forces. For instance, the maximum Young's modulus for $P$. pectinatus was 116.23 MPa, whereas for $P$. crispus, the highest value was four times lower $(26.60 \mathrm{MPa})$. The present study supplements an aquatic plant biomechanics database that has been created in recent years.
\end{abstract}

Keywords: curlyleaf pondweed, sago pondweed, mechanical traits, environmental management, aquatic macrophytes, bending, tension

*e-mail: aloboda@igf.edu.pl 


\section{Introduction}

As stated by Nepf [1], the trade-off between floods and ecological management underlines the need for a reliable method to predict channel resistance in the presence of vegetation. The need for improving flow-vegetationsediment interactions is important for engineering (e.g., hydraulic resistance estimation) [2-5] and ecological (e.g., stream restoration) [6-8] applications. Recently there has been an increased interest in management and control methods of invasive aquatic plants that have played an important role in river colonization during recent years [9-10 and literature therein]. Researchers have mostly concentrated on plant systematics or hybridization [e.g., 11] rather than on the basic biology, which should allow for precise parameterization of the interaction between aquatic plants and physical processes in rivers [12]. From this point of view, plant biomechanics seems to be an important property that should be regularly monitored, especially for invasive and phenoplastic aquatic plants, which have strong community dynamics. However, not all freshwater species behave in the same way, and most of them - even those belonging to the same family - have completely different vegetative periods and reproductive methods that are often associated with their adaptation to seasonally changeable abiotic factors [13]. Many of these factors, including temperature or the availability of light and water nutrients, have been extensively studied [e.g., 14-15]. For instance, Riis et al. [14] claimed that aquatic macrophytes spread differently in relation to changing conditions (i.e., water temperature or light availability). In addition, as stated by de los Santos et al. [15], variations in plant biomechanics may be due to changes in water chemistry. However, only recently did Miler et al. [16] investigate the seasonal changes in the biomechanical properties of some aquatic plants (i.e., Glyceria spp., Fontinalis spp., Ranunculus spp., and Myriophyllum spp.). However, the authors did not find a general tendency in the seasonal changes of cross-sectional area, diameter, or biomechanical traits like 'tension' Young's modulus or elasticity in the studied plants. They claimed that these

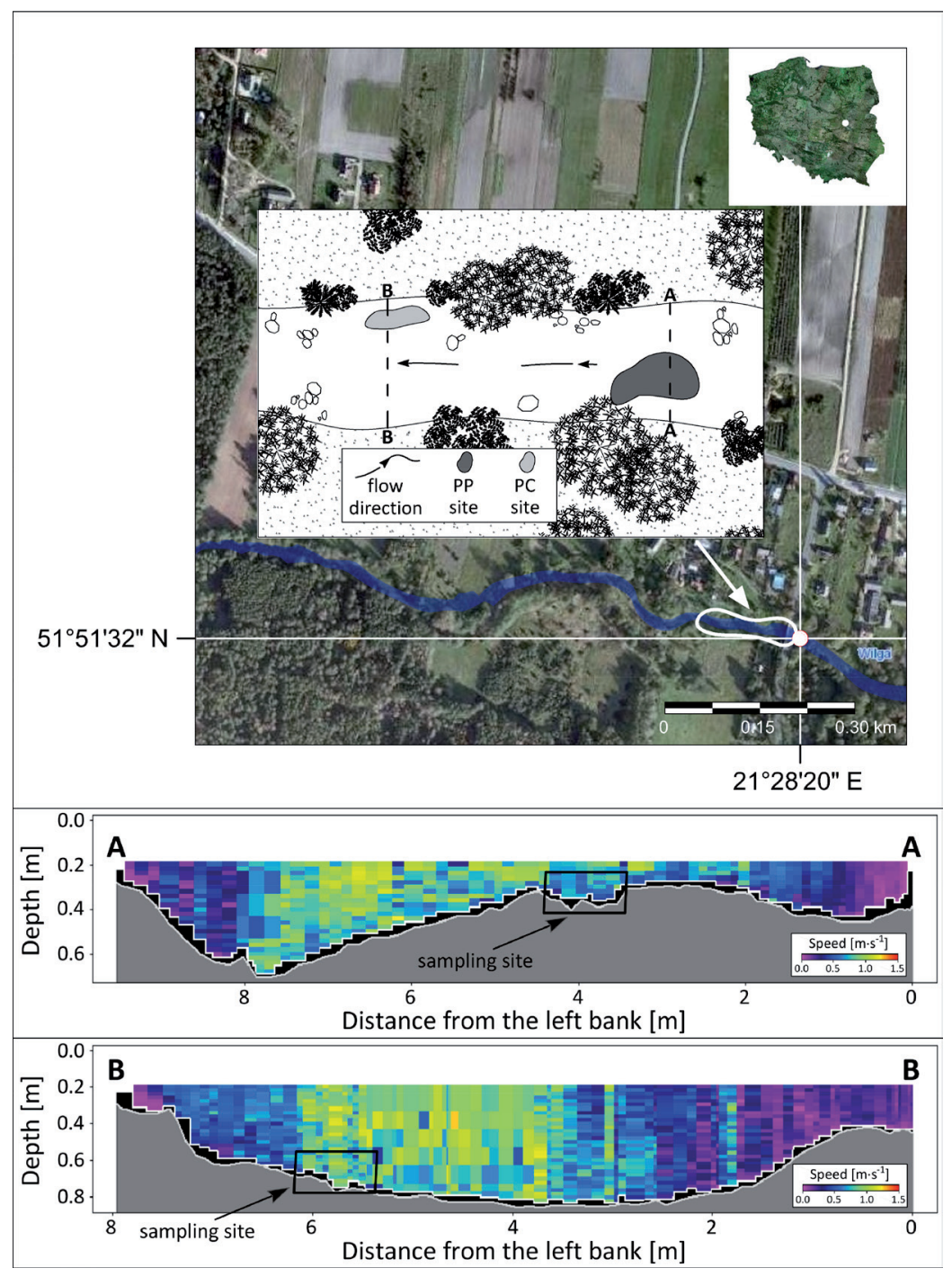

Fig. 1. Sampling site on the Wilga River with the velocity spatial distribution on channel sections of P. pectinatus (PP) and P. crispus (PC) measured using an acoustic Doppler current profiler. 
plants did not use an increase in mechanical strength and/or stem flexibility as an adaptive strategy to resist harsh conditions during winter/spring.

This paper investigates the seasonal changes in biomechanical traits of two Potamogeton spp. that belong to a common submerged perennial herb [17]. These plants occur in rivers, lakes, and marshes around the world [18-19]. P pectinatus is characterised by slender round shoots up to $3 \mathrm{~m}$ long with narrow linear leaves, whereas P. crispus has shorter heavily branched stems up to $2 \mathrm{~m}$ [17]. Both plants can reproduce vegetatively $[18,20]$. The significant quality of these plants is a very successful survival strategy that contributes to their wide distribution [18, 21]. Moreover, the important dissimilarity that distinguishes $P$. crispus from other aquatic macrophytes is its unique life cycle. Firstly, the plants flower in spring, then die back or senesce in early summer. Finally, in autumn, the plants start to sprout again, given appropriate conditions in terms of light and water temperature [20]. Such phenological behaviour should be naturally considered with changes in biomechanical properties of these plants, which have only been investigated by a small number of researchers $[16,22-23]$ without any attention paid to the seasonal changes.

The first goal of this paper is to show detailed information about the seasonal changes in the biomechanical traits of the stems of two submerged aquatic plants, $P$. pectinatus. and $P$. crispus, which live together in the Wilga River in Poland. The second goal is to supplement an aquatic plant biomechanics database that should have a strong impact on the ecological management of aquatic ecosystems. Moreover, we address two hypotheses:

1. The trends in changes in biomechanical properties of Potamogeton spp. differ between species at the same moment in phenological time due to the changes in their morphological structure and may be independent of their stage of growth.

2. The changes in hydrological conditions are closely related to changes in biomechanical parameters of Potamogeton spp., and they are independent of the plant species.

\section{Materials and Methods}

\section{Sampling Sites and Data Collection}

The studied plants, $P$. pectinatus and $P$. crispus, were collected in 2016 and 2017 over the course of the entire plant growth season. The sampling site $\left(51^{\circ} 51^{\prime} 30^{\prime \prime} \mathrm{N}\right.$, $21^{\circ} 28^{\prime} 20^{\prime \prime}$ E, Fig. 1) is located $60 \mathrm{~km}$ south of Warsaw, Poland, on the Wilga River. It is the right-bank tributary of the Vistula River, in which the channel bed consists of small stones and gravel with sand [24]. This location was chosen due to a common occurrence of two species from the family Potamogetonaceae with contrasting hydrological characteristics of plant locations in the river.

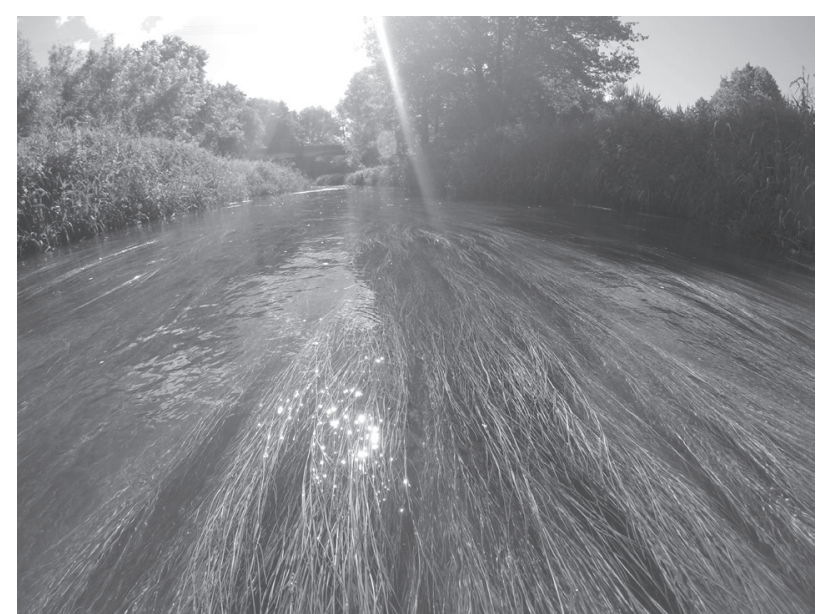

Fig. 2. The Wilga River with the view at the P. pectinatus sampling site.

In 2016 P. pectinatus was collected six times from May to October. The vegetation season in 2017 started in June and individuals were collected until November. After these periods, there were no visible macroscopic living specimens in the river, or the plants were damaged enough that they were not suitable for biomechanical measurements. The plants covered the greater part of the riverbed (Fig. 2), and samples were collected from the middle section of the channel, where the flow was around $0.5-0.7 \mathrm{~m} \cdot \mathrm{s}^{-1}$ (Fig. 1). The sediment consisted of medium, moderately well sorted sand $\left(\mathrm{D}_{16}=0.29 \mathrm{~mm}\right.$, $\mathrm{D}_{50}=0.46 \mathrm{~mm}, \mathrm{D}_{84}=1.97 \mathrm{~mm}$, Fig. 3). The second investigated species, P. crispus, was found and collected eight times in 2016 from May to December. In 2017 the plant was found and collected at the same sampling site only twice, in August and November. The habitat of this hydrophyte is located near the right river bank, covered by medium, poorly sorted sand $\left(\mathrm{D}_{16}=0.28 \mathrm{~mm}, \mathrm{D}_{50}=0.45 \mathrm{~mm}, \mathrm{D}_{84}=15.66 \mathrm{~mm}\right.$; Fig. 3), where the flow is distinctly higher $\left(1.0 \mathrm{~m} \cdot \mathrm{s}^{-1} ;\right.$ Fig. 1$)$ than at the collection site of $P$. pectinatus. The high velocities and water discharge and the accompanying high water levels in the habitat of this plant made it impossible to collect this hydrophyte in May and October 2017 (Fig. 4).

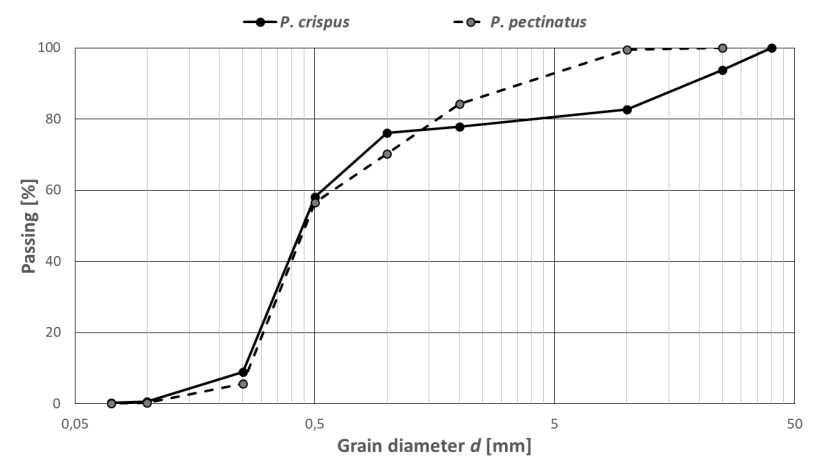

Fig. 3. Granulometric distribution curves of bed sediment from sampling sites of $P$. pectinatus and P. crispus. 
a)

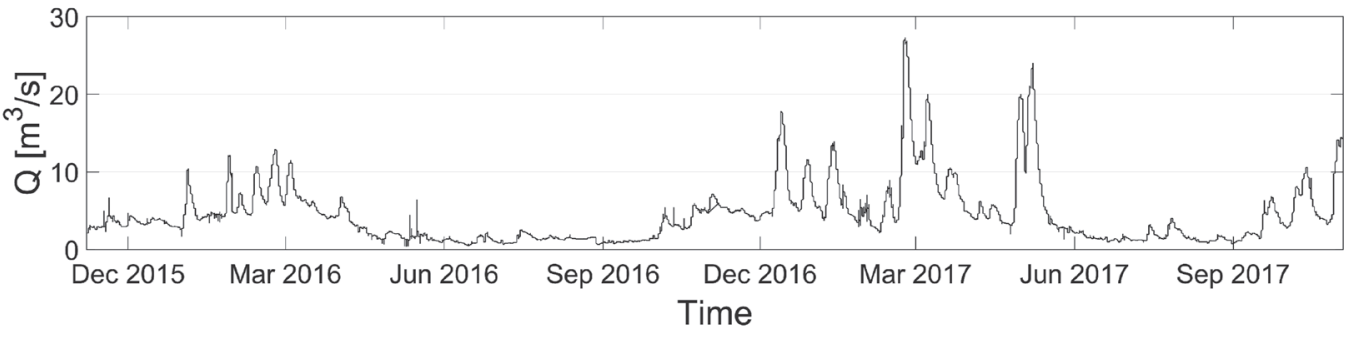

b)

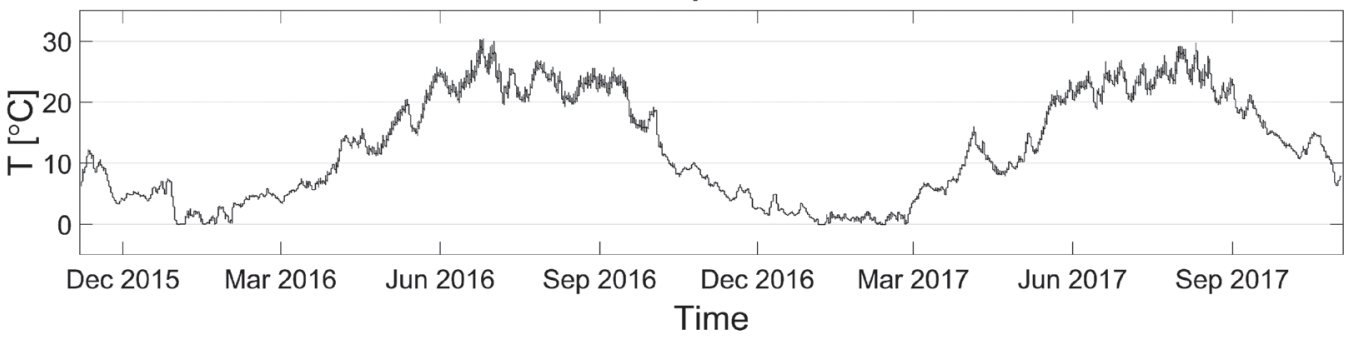

c)

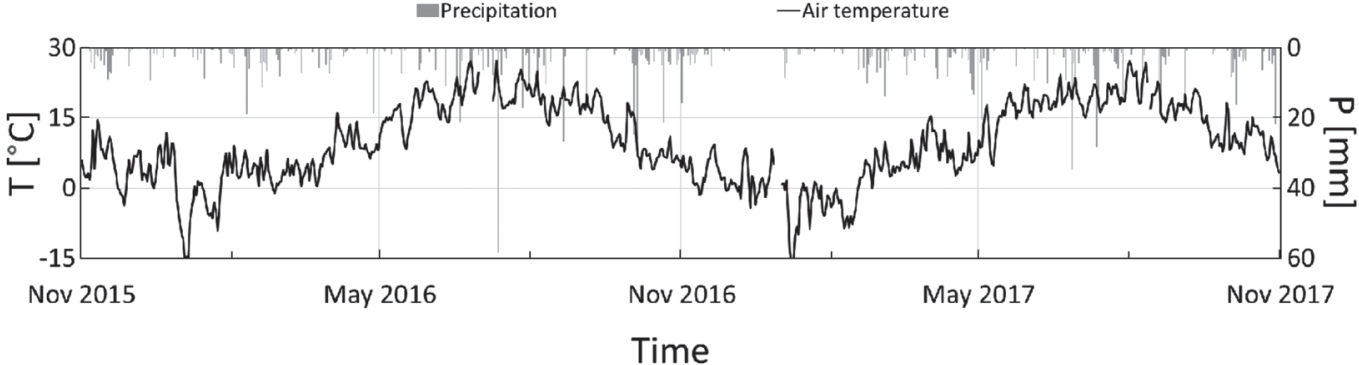

Fig. 4. Time series showing the following: the discharge Q over a 2-year period in the Świder River a), the temperature T over a 2-year period in the Vistula River b), and the daily mean air temperature T and precipitation P over a 2-year period in the Świder c).

In order to better understand the phenomenon of changes in plant biomechanics we analysed meteorological and hydrological data. Due to the lack of a gauging station on the Wilga, continuous hydrological data sets are not available. However, located close to the Wilga is the Świder River, which has very similar hydrological and morphological characteristics. An analysis of the hydrological data from gauges located in the Vistula and Swider Rivers (Fig. 4a-b) and supplementary meteorological data (Fig. 4c) allows for estimating the hydrological regime of the Wilga over a 2-year period of measurements. In addition, the water temperature, dissolved oxygen (DO), $\mathrm{pH}$, and specific conductance (SPC) on the day of plant collection were measured 'in-situ' in the second season with the use of a Professional Plus (Pro Plus) multiparameter instrument (YSI, Yellow Springs, USA). The time series of the daily mean air temperature and precipitation from the meteorological station in the Swider were also investigated. Furthermore, the bathymetry and velocity spatial distribution of the Wilga in the cross-section of the sampling site were obtained with use of an acoustic Doppler current profiler (ADCP), RiverSurveyor S5 model (manufactured by SonTek, San Diego, USA).

\section{Equipment and Plant Preparation}

Transportation of plants involved their temporary placement in a 52-L plastic aquarium equipped with an aeration device. While in the laboratory, plants were stored in a 112-L tank. Natural conditions were mimicked by inclusion of two water pumps, a standard aeration device, and a fluorescent lamp with two light bulbs that were illuminated up to $12 \mathrm{~h}$ per day. During each measurement campaign, the photographs of individual plants and their cross-sectional areas were taken prior to biomechanical tests to define the morphology characteristics and internal structure of these aquatic macrophytes (Figs 5-6).

Biomechanical tests consisted of three-point bending and tension tests using a Tinius Olsen Bench Top Testing Machine, 5ST Model. The first step before testing was appropriate sample preparation. After removing individuals from the aquarium, the plant stem was cut into $7 \mathrm{~cm}$ pieces. The diameter of each part was measured with the use of a microscope or calliper. In tension tests, sample preparation included gluing short strips of sandpaper to the ends of the samples to prevent them from slipping from the machine clamps [25]. During measurements, the samples were submerged 
in water (wet testing conditions) to avoid the drying of stems [details in 25]. Therefore, only a few samples were prepared at once to minimize the time that the plant was outside the aquatic environment.

\section{Biomechanical Tests}

The measurement results were processed with Horizon software included with the device. Three-point bending tests include the outcomes of maximum deflection, maximum stress (force per area), and maximum force, which is the load at the given point of maximum deflection. The wet testing conditions affected the final values of the mechanical characteristics, which were reduced by the buoyancy forces. This step was not applied in tension tests due to a negligible influence on results. The calculated bending quantities included flexural rigidity, flexural modulus, and flexural strain. The calculations were made using the appropriate formulas [26-27]:

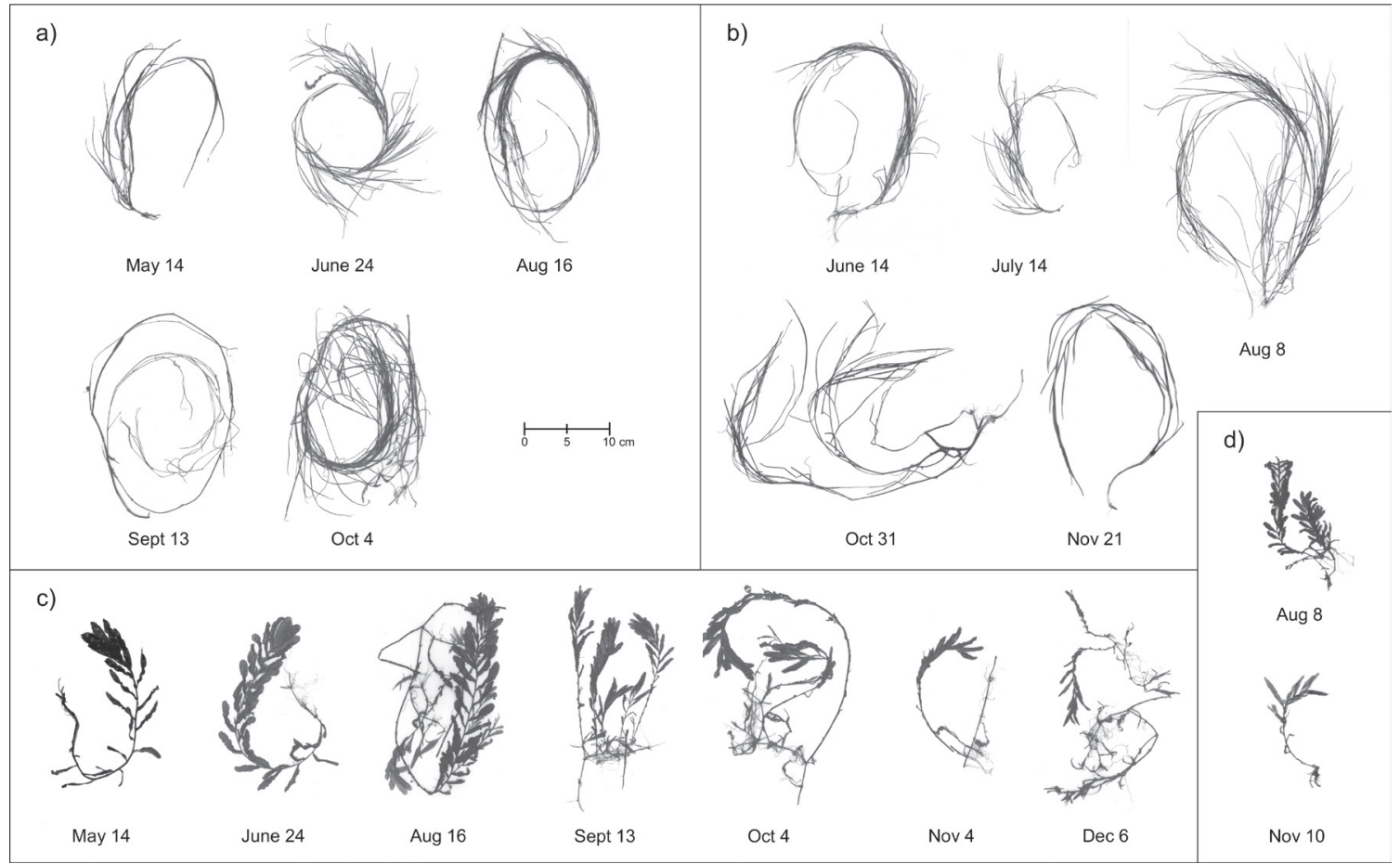

Fig. 5. Photographs of tested P. pectinatus L. in 2016 a) and 2017 b); and P. crispus L. in 2016 c) and 2017 d) at different time points.

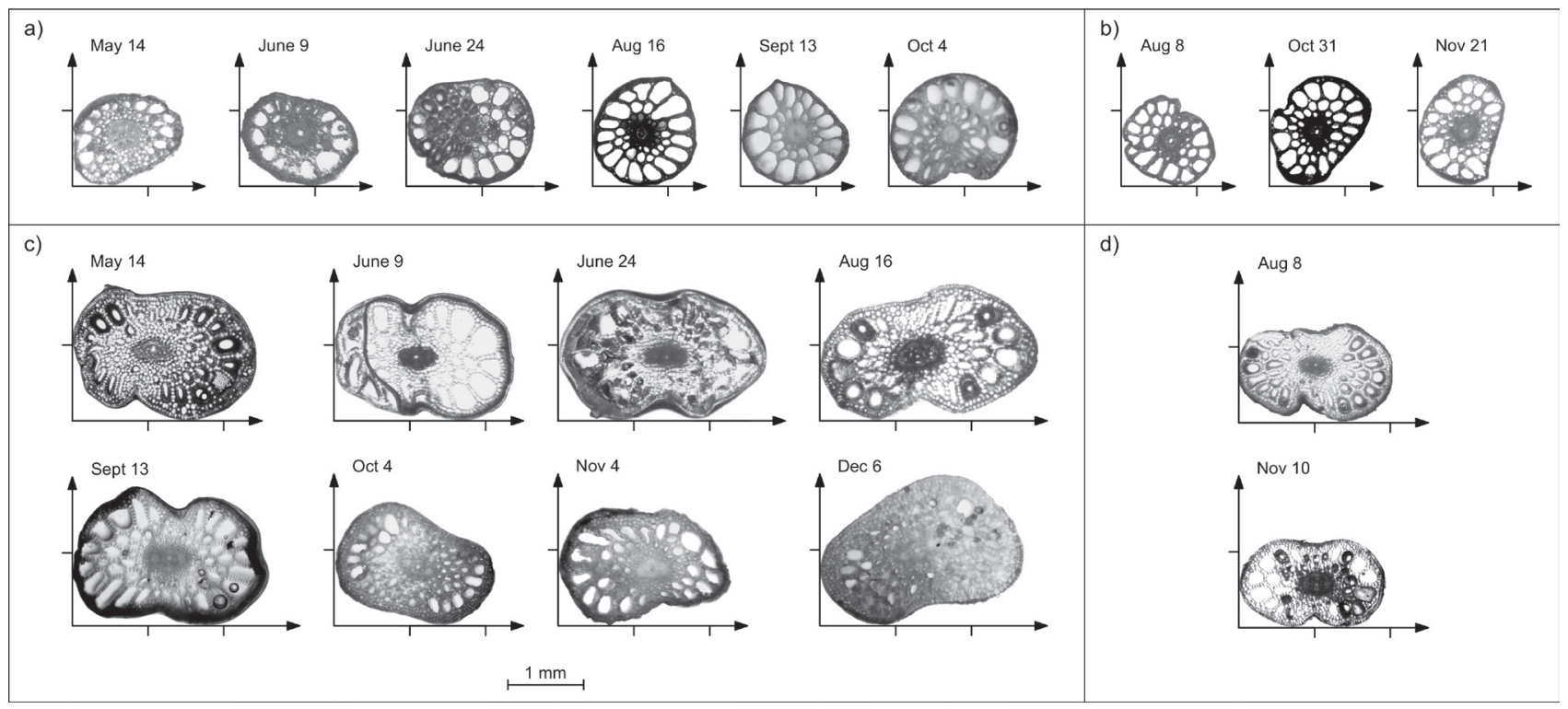

Fig. 6. Cross-sections of P. pectinatus L. in 2016 a) and 2017 b); and P. crispus L. in 2016 c) and 2017 d) in all time periods of plant collection. 
- Flexural rigidity $E I: E I=\frac{S^{3} F}{48 \Delta}$, where $S$ is the span of the stem between the support bars [mm], $F$ describes the force at a given point on the initial linear elastic part of the force-deflection curve $[\mathrm{N}]$, and $\Delta$ stands for deflection [mm].

- Flexural modulus $E_{F}: E_{F}=\frac{E I}{I}$, where $E I$ is flexural rigidity $\left[\mathrm{N} \cdot \mathrm{mm}^{2}\right]$ and $I$ stands for the second moment of area defined as $I_{C}=\frac{\pi D^{4}}{64},\left[\mathrm{~mm}^{4}\right]$ for $P$. pectinatus, circular shape; and $I_{E}=\frac{\pi R r^{3}}{4},\left[\mathrm{~mm}^{4}\right]$ for P. crispus, elliptical shape $(D$ is the cross-sectional diameter, [mm], $R$ is the long radius, and $r$ is the short radius of the ellipse cross-section [mm]).

- Flexural strain $\varepsilon_{f}: \quad \varepsilon_{f}=\frac{6 \Delta_{\max } D}{S^{2}}$, where $\Delta_{\max }$ is maximum deflection $[\mathrm{mm}], \stackrel{S^{2}}{D}$ is the diameter of the stem, and $S$ stands for the span of the stem between the support bars [mm]. Tension tests were performed until the complete failure of a specimen. The Horizon software recorded data of breaking values of force, stress, and strain (maximum values at the rupture of the stem). These characteristics were needed to calculate the modulus of elasticity, i.e. Young's modulus, using formula [26]:

- Young's modulus $E_{T}: E_{T}=\frac{\sigma}{\varepsilon}$, where $\sigma$ is the tensile stress $[\mathrm{MPa}]$ and $\varepsilon$ stands for the strain [-].

\section{Statistical Analysis}

The bending outcomes of plant biomechanics, according to seasonal changes, were investigated through maximum force, flexural rigidity, flexural modulus, and maximum deflection, and the tensile characteristics, i.e., breaking force and Young's modulus. To analyse the differences in mean values of the biomechanical parameters between each period of plant measurements, a statistical analysis was performed using one of the resampling tests, namely the permutation test [28]. Moreover, the analysis of biomechanical traits between similar periods in seasons was also performed. For the null hypothesis, it was assumed that the mean equality contrary to the alternative hypothesis of significant differences in means between two groups of data describing an individual period of measurements. Then, the average difference module in both datasets was counted. Moreover, to find the significance of differences, two additional steps were carried out, the random mixing of values in both groups of data, keeping their numbers, and the calculation of the amount of difference for the new datasets. Further, to understand which values could be accepted when the two datasets did not differ, it was necessary to repeat the above steps. The number of 100,000 groups was considered due to the large amount of total groups. To calculate the p-value, the percentage of mean differences for random fluctuations greater than observed was taken. The differences between groups of data are statistically significant when the the p-value is lower than 0.05 .

\section{Results}

\section{Hydrological and Meteorological Data}

In 2016, the water temperature ranged from $2.3^{\circ} \mathrm{C}$ on 6 December to $24.0^{\circ} \mathrm{C}$ on 24 June (Table 1). The measured water discharge was at a maximum $\left(3.62 \mathrm{~m}^{3} \cdot \mathrm{s}^{-1}\right)$ on 4 November, while the minimum value $\left(0.62 \mathrm{~m}^{3} \cdot \mathrm{s}^{-1}\right)$ was observed on 13 September (Table 1). In comparison to the first season, in 2017, the water temperature values varied from $4.5^{\circ} \mathrm{C}$ on 21 November to $23.5^{\circ} \mathrm{C}$ on 12 July (Table 1 ). The maximum water discharge $\left(6.68 \mathrm{~m}^{3} \cdot \mathrm{s}^{-1}\right)$ was observed on 31 October, whereas the minimum value $\left(0.76 \mathrm{~m}^{3} \cdot \mathrm{s}^{-1}\right)$ was measured on 12 July (Table 1). Moreover, during the second season, the water quality parameters were measured, i.e., dissolved oxygen (DO), $\mathrm{pH}$, and specific conductance (SPC). The obtained outcomes were in line with those obtained within the continuous measurement done by Rajwa-Kuligiewicz et al. [29], although the DO concentration varied from 4.28 to $9.21 \mathrm{mg} \cdot \mathrm{L}^{-1}$ during different periods of plant collection with the extreme value of $22.54 \mathrm{mg} \cdot \mathrm{L}^{-1}$ associated with very low water temperature on 21 November. The $\mathrm{pH}$ values ranged from 7.51 to 7.80 , showing slightly alkaline water, while the conductivity oscillated around $550 \mu \mathrm{S} \cdot \mathrm{cm}^{-1}$.

In relation to the annual hydrological data, in 2016, high values of discharge (Fig. 4a) were observed during the winter and early spring, whereas later in summer it stabilised at low values (approximately $0.6 \mathrm{~m}^{3} \cdot \mathrm{s}^{-1}$; Fig. 4a). This period was characterised by rather steady flow conditions without major changes.

Table 1. Temperature (T) and discharge (Q) for the Wilga River for all time periods of plant collection

\begin{tabular}{|c|c|c|}
\hline Day of sample collection & $\mathrm{T}\left({ }^{\circ} \mathrm{C}\right)$ & $\mathrm{Q}\left(\mathrm{m}^{3} \cdot \mathrm{s}^{-1}\right)$ \\
\hline 14 May & 17.2 & 1.01 \\
\hline 9 June & 20.0 & 0.63 \\
\hline 24 June & 24.1 & 0.97 \\
\hline 16 Aug. & 17.7 & 0.91 \\
\hline 13 Sept. & 20.4 & 0.62 \\
\hline 4 Oct. & 15.7 & 1.47 \\
\hline 4 Nov. & 6.0 & 3.62 \\
\hline 6 Dec. & 2.3 & 2.57 \\
\hline 7 June & 17.9 & 1.10 \\
\hline 14 June & 14.9 & 0.89 \\
\hline 12 July & 23,5 & 0.76 \\
\hline 8 Aug. & 16.9 & 0.79 \\
\hline 31 Oct. & 5.0 & 6,68 \\
\hline 10 Nov. & 7.9 & 3.12 \\
\hline 21 Nov. & 4.5 & 3.23 \\
\hline
\end{tabular}


Table 2. Diameter and three-point bending test results for all periods of measurements of P. pectinatus and P. crispus in 2016.

\begin{tabular}{|c|c|c|c|c|c|c|c|c|c|}
\hline \multicolumn{10}{|c|}{ P. pectinatus } \\
\hline \multirow{2}{*}{\multicolumn{2}{|c|}{ Parameter }} & \multicolumn{2}{|c|}{14 May } & \multicolumn{2}{|c|}{9 June } & \multicolumn{2}{|c|}{24 June } & \multicolumn{2}{|c|}{16 Aug. } \\
\hline & & $\begin{array}{l}\text { Mean } \\
\pm \text { S.D. }\end{array}$ & Median & $\begin{array}{l}\text { Mean } \\
\pm \text { S.D. }\end{array}$ & Median & $\begin{array}{l}\text { Mean } \\
\pm \text { S.D. }\end{array}$ & Median & $\begin{array}{l}\text { Mean } \\
\pm \text { S.D. }\end{array}$ & Median \\
\hline \multicolumn{2}{|c|}{ Number of samples } & \multicolumn{2}{|l|}{20} & \multicolumn{2}{|l|}{20} & \multicolumn{2}{|l|}{18} & \multicolumn{2}{|l|}{19} \\
\hline Diameter & $\mathrm{mm}$ & $1.30 \pm 0.31$ & 1.21 & $0.97 \pm 0.23$ & 0.88 & $1.37 \pm 0.29$ & 1.32 & $1.56 \pm 0.44$ & 1.45 \\
\hline Maximum force & $\mathrm{N}$ & $0.022 \pm 0.013$ & 0.020 & $0.014 \pm 0.010$ & 0.014 & $0.035 \pm 0.044$ & 0.027 & $0.029 \pm 0.026$ & 0.017 \\
\hline Maximum stress & $\mathrm{MPa}$ & $0.019 \pm 0.015$ & 0.012 & $0.023 \pm 0.020$ & 0.020 & $0.021 \pm 0.013$ & 0.018 & $0.013 \pm 0.007$ & 0.014 \\
\hline Flexural strain & $\%$ & $2.94 \pm 1.91$ & 2.15 & $3.74 \pm 1.97$ & 4.18 & $5.57 \pm 2.12$ & 5.91 & $5.53 \pm 2.78$ & 6.67 \\
\hline Max. deflection & $\mathrm{mm}$ & $8.41 \pm 5.40$ & 5.12 & $14.44 \pm 6.63$ & 18.85 & $14.92 \pm 4.82$ & 13.27 & $12.70 \pm 5.99$ & 13.89 \\
\hline Sec. m. of area & $\mathrm{mm}^{4}$ & $0.196 \pm 0.222$ & 0.104 & $0.063 \pm 0.092$ & 0.029 & $0.225 \pm 0.206$ & 0.149 & $0.435 \pm 0.456$ & 0.217 \\
\hline Flexural rigidity & $\mathrm{N} \cdot \mathrm{mm}^{2}$ & $10.63 \pm 7.77$ & 8.19 & $3.29 \pm 3.42$ & 2.21 & $12.01 \pm 8.12$ & 9.83 & $21.09 \pm 19.27$ & 15.72 \\
\hline Flexural modulus & $\mathrm{MPa}$ & $94.51 \pm 84.66$ & 57.82 & $86.59 \pm 80.25$ & 61.40 & $90.18 \pm 79.13$ & 55.72 & $94.09 \pm 120.77$ & 44.63 \\
\hline \multirow{2}{*}{\multicolumn{2}{|c|}{ Parameter }} & \multicolumn{2}{|c|}{13 Sept. } & \multicolumn{2}{|c|}{4 Oct. } & \multicolumn{2}{|c|}{4 Nov. } & \multicolumn{2}{|c|}{6 Dec. } \\
\hline & & $\begin{array}{l}\text { Mean } \\
\pm \text { S.D. }\end{array}$ & Median & $\begin{array}{l}\text { Mean } \\
\pm \text { S.D. }\end{array}$ & Median & $\begin{array}{l}\text { Mean } \\
\pm \text { S.D. }\end{array}$ & Median & $\begin{array}{l}\text { Mean } \\
\pm \text { S.D. }\end{array}$ & Median \\
\hline \multicolumn{2}{|c|}{ Number of samples } & \multicolumn{2}{|l|}{15} & \multicolumn{2}{|l|}{19} & \multicolumn{2}{|l|}{ - } & \multicolumn{2}{|l|}{-} \\
\hline Diameter & $\mathrm{mm}$ & $1.68 \pm 0.42$ & 1.58 & $1.26 \pm 0.33$ & 1.24 & - & - & - & - \\
\hline Maximum force & $\mathrm{N}$ & $0.030 \pm 0.025$ & 0.023 & $0.024 \pm 0.029$ & 0.018 & - & - & - & - \\
\hline Maximum stress & $\mathrm{MPa}$ & $0.013 \pm 0.008$ & 0.012 & $0.019 \pm 0.016$ & 0.016 & - & - & - & - \\
\hline Flexural strain & $\%$ & $6.64 \pm 1.81$ & 6.42 & $4.91 \pm 2.91$ & 5.80 & - & - & - & - \\
\hline Max. deflection & $\mathrm{mm}$ & $15.32 \pm 4.86$ & 17.79 & $13.94 \pm 7.11$ & 19.29 & - & - & - & - \\
\hline Sec. m. of area & $\mathrm{mm}^{4}$ & $0.531 \pm 0.456$ & 0.306 & $0.178 \pm 0.198$ & 0.116 & - & - & - & - \\
\hline Flexural rigidity & $\mathrm{N} \cdot \mathrm{mm}^{2}$ & $12.65 \pm 10.53$ & 6.06 & $5.31 \pm 5.86$ & 2.72 & - & - & - & - \\
\hline Flexural modulus & $\mathrm{MPa}$ & $36.66 \pm 31.63$ & 25.75 & $55.08 \pm 75.78$ & 23.47 & - & - & - & - \\
\hline \multicolumn{10}{|c|}{ P. crispus } \\
\hline \multirow{2}{*}{\multicolumn{2}{|c|}{ Parameter }} & \multicolumn{2}{|c|}{14 May } & \multicolumn{2}{|c|}{9 June } & \multicolumn{2}{|c|}{24 June } & \multicolumn{2}{|c|}{16 Aug. } \\
\hline & & $\begin{array}{l}\text { Mean } \\
\pm \text { S.D. }\end{array}$ & Median & $\begin{array}{l}\text { Mean } \\
\pm \text { S.D. }\end{array}$ & Median & $\begin{array}{l}\text { Mean } \\
\pm \text { S.D. }\end{array}$ & Median & $\begin{array}{l}\text { Mean } \\
\pm \text { S.D. }\end{array}$ & Median \\
\hline Number of sam & aples & 20 & & 20 & & 20 & & 20 & \\
\hline Diameter & $\mathrm{mm}$ & $2.10 \pm 0.37$ & 2.09 & $2.43 \pm 0.17$ & 2.47 & $1.87 \pm 0.37$ & 1.85 & $1.97 \pm 0.40$ & 2.04 \\
\hline Maximum force & $\mathrm{N}$ & $0.071 \pm 0.039$ & 0.069 & $0.069 \pm 0.031$ & 0.063 & $0.057 \pm 0.033$ & 0.048 & $0.081 \pm 0.044$ & 0.070 \\
\hline Maximum stress & $\mathrm{MPa}$ & $0.022 \pm 0.012$ & 0.018 & $0.015 \pm 0.006$ & 0.014 & $0.021 \pm 0.010$ & 0.022 & $0.026 \pm 0.010$ & 0.026 \\
\hline Flexural strain & $\%$ & $7.81 \pm 3.11$ & 8.00 & $8.64 \pm 2.68$ & 8.24 & $6.64 \pm 2.07$ & 6.91 & $6.48 \pm 1.92$ & 6.04 \\
\hline Max. deflection & $\mathrm{mm}$ & $13.63 \pm 4.50$ & 14.43 & $13.17 \pm 4.06$ & 13.07 & $12.99 \pm 3.25$ & 13.15 & $12.18 \pm 2.66$ & 11.97 \\
\hline Sec. m. of area & $\mathrm{mm}^{4}$ & $1.126 \pm 0.785$ & 0.928 & $1.753 \pm 0.445$ & 1.828 & $0.737 \pm 0.508$ & 0.576 & $0.919 \pm 0.709$ & 0.842 \\
\hline Flexural rigidity & $\mathrm{N} \cdot \mathrm{mm}^{2}$ & $25.79 \pm 17.07$ & 22.11 & $36.13 \pm 24.54$ & 29.70 & $25.42 \pm 15.04$ & 21.16 & $65.67 \pm 34.17$ & 62.97 \\
\hline Flexural modulus & $\mathrm{MPa}$ & $33.54 \pm 34.53$ & 21.80 & $19.78 \pm 11.91$ & 16.65 & $51.59 \pm 34.68$ & 41.55 & $105.21 \pm 75.66$ & 82.40 \\
\hline
\end{tabular}


Table 2. Continued.

\begin{tabular}{|c|c|c|c|c|c|c|c|c|c|}
\hline \multirow{2}{*}{ Parameter } & \multicolumn{2}{|c|}{13 Sept. } & \multicolumn{2}{c|}{ 4 Oct. } & \multicolumn{2}{c|}{ 4 Nov. } & \multicolumn{2}{c|}{6 Dec. } \\
\cline { 3 - 11 } & $\begin{array}{c}\text { Mean } \\
\pm \text { S.D. }\end{array}$ & Median & $\begin{array}{c}\text { Mean } \\
\pm \text { S.D. }\end{array}$ & Median & $\begin{array}{c}\text { Mean } \\
\pm \text { S.D. }\end{array}$ & $\begin{array}{c}\text { Median } \\
\pm \text { Mean } \\
\pm \text { S.D. }\end{array}$ & $\begin{array}{c}\text { Median } \\
20\end{array}$ \\
\hline Number of samples & \multicolumn{2}{|c|}{20} & \multicolumn{2}{c|}{19} & \multicolumn{2}{c|}{20} & 20 \\
\hline Diameter & $\mathrm{mm}$ & $2.23 \pm 0.36$ & 2.37 & $2.21 \pm 0.41$ & 2.28 & $1.84 \pm 0.27$ & 1.83 & $1.70 \pm 0.24$ & 1.65 \\
\hline Maximum force & $\mathrm{N}$ & $0.059 \pm 0.028$ & 0.061 & $0.097 \pm 0.078$ & 0.069 & $0.050 \pm 0.020$ & 0.052 & $0.034 \pm 0.018$ & 0.031 \\
\hline Maximum stress & $\mathrm{MPa}$ & $0.014 \pm 0.006$ & 0.016 & $0.023 \pm 0.011$ & 0.019 & $0.019 \pm 0.007$ & 0.019 & $0.015 \pm 0.007$ & 0.014 \\
\hline Flexural strain & $\%$ & $9.57 \pm 2.67$ & 10.19 & $8.18 \pm 1.75$ & 8.63 & $7.28 \pm 1.72$ & 6.88 & $6.68 \pm 2.02$ & 6.18 \\
\hline Max. deflection & $\mathrm{mm}$ & $15.57 \pm 2.90$ & 15.33 & $13.88 \pm 2.73$ & 13.82 & $14.66 \pm 3.23$ & 14.73 & $14.40 \pm 3.54$ & 14.07 \\
\hline Sec. m. of area & $\mathrm{mm}$ & $1.383 \pm 0.672$ & 1.536 & $1.363 \pm 0.776$ & 1.327 & $0.640 \pm 0.404$ & 0.551 & $0.457 \pm 0.289$ & 0.360 \\
\hline Flexural rigidity & $\mathrm{N} \cdot \mathrm{mm}{ }^{2}$ & $46.28 \pm 40.97$ & 27.12 & $46.93 \pm 45.79$ & 28.42 & $31.55 \pm 24.58$ & 25.71 & $18.41 \pm 16.25$ & 10.51 \\
\hline Flexural modulus & $\mathrm{MPa}$ & $36.38 \pm 27.46$ & 33.08 & $43.17 \pm 57.69$ & 28.74 & $55.22 \pm 41.36$ & 48.99 & $52.71 \pm 62.24$ & 26.04 \\
\hline
\end{tabular}

Table 3. Diameter and three-point bending test results for all periods of measurement of P. pectinatus and P. crispus in 2017

\begin{tabular}{|c|c|c|c|c|c|c|c|c|c|}
\hline \multicolumn{10}{|c|}{ P. pectinatus } \\
\hline \multirow{2}{*}{\multicolumn{2}{|c|}{ Parameter }} & \multicolumn{2}{|c|}{14 June } & \multicolumn{2}{|c|}{12 July } & \multicolumn{2}{|c|}{8 Aug. } & \multicolumn{2}{|c|}{31 Oct. } \\
\hline & & $\begin{array}{l}\text { Mean } \\
\pm \text { S.D. }\end{array}$ & Median & $\begin{array}{l}\text { Mean } \\
\pm \text { S.D. }\end{array}$ & Median & $\begin{array}{l}\text { Mean } \\
\pm \text { S.D. }\end{array}$ & Median & $\begin{array}{c}\text { Mean } \\
\pm \text { S.D. }\end{array}$ & Median \\
\hline \multicolumn{2}{|c|}{ Number of samples } & \multicolumn{2}{|l|}{19} & \multicolumn{2}{|l|}{19} & \multicolumn{2}{|l|}{19} & \multicolumn{2}{|l|}{29} \\
\hline Diameter & $\mathrm{mm}$ & $1.22 \pm 0.15$ & 1.20 & $0.84 \pm 0.20$ & 0.80 & $1.21 \pm 0.20$ & 1.23 & $1.24 \pm 0.33$ & 1.12 \\
\hline Maximum force & $\mathrm{N}$ & $0.028 \pm 0.009$ & 0.028 & $0.019 \pm 0.008$ & 0.020 & $0.025 \pm 0.009$ & 0.025 & $0.022 \pm 0.016$ & 0.016 \\
\hline Maximum stress & $\mathrm{MPa}$ & $0.024 \pm 0.007$ & 0.023 & $0.037 \pm 0.020$ & 0.034 & $0.023 \pm 0.011$ & 0.021 & $0.017 \pm 0.006$ & 0.016 \\
\hline Flexural strain & $\%$ & $5.30 \pm 0.50$ & 5.22 & $3.49 \pm 1.14$ & 3.25 & $5.04 \pm 0.93$ & 4.92 & $4.67 \pm 1.42$ & 4.66 \\
\hline Max. deflection & $\mathrm{mm}$ & $16.14 \pm 1.51$ & 16.04 & $15.32 \pm 3.69$ & 16.22 & $15.41 \pm 2.34$ & 15.84 & $14.03 \pm 3.19$ & 15.21 \\
\hline Sec. m. of area & $\mathrm{mm}^{4}$ & $0.119 \pm 0.081$ & 0.102 & $0.035 \pm 0.053$ & 0.020 & $0.124 \pm 0.093$ & 0.112 & $0.182 \pm 0.255$ & 0.077 \\
\hline Flexural rigidity & $\mathrm{N} \cdot \mathrm{mm}^{2}$ & $16.53 \pm 5.29$ & 16.44 & $5.93 \pm 8.26$ & 3.74 & $9.37 \pm 2.94$ & 9.70 & $11.52 \pm 7.82$ & 8.70 \\
\hline Flexural modulus & $\mathrm{MPa}$ & $168.50 \pm 77.21$ & 171.86 & $252.97 \pm 236.87$ & 151.00 & $109.72 \pm 75.91$ & 90.76 & $117.56 \pm 73.94$ & 99.57 \\
\hline & & \multicolumn{2}{|c|}{ P. pectinatus } & \multicolumn{4}{|c|}{ P. crispus } & & \\
\hline \multirow{2}{*}{\multicolumn{2}{|c|}{ Parameter }} & \multicolumn{2}{|c|}{21 Nov. } & \multicolumn{2}{|l|}{8 Aug. } & \multicolumn{2}{|c|}{10 Nov. } & & \\
\hline & & $\begin{array}{l}\text { Mean } \\
\pm \text { S.D. }\end{array}$ & Median & $\begin{array}{l}\text { Mean } \\
\pm \text { S.D. }\end{array}$ & Median & $\begin{array}{l}\text { Mean } \\
\pm \text { S.D. }\end{array}$ & Median & & \\
\hline \multicolumn{2}{|c|}{ Number of samples } & & & \multicolumn{2}{|l|}{20} & \multicolumn{2}{|l|}{16} & & \\
\hline Diameter & $\mathrm{mm}$ & $1.17 \pm 0.23$ & 1.12 & $1.90 \pm 0.40$ & 2.01 & $1.65 \pm 0.26$ & 1.62 & & \\
\hline Maximum force & $\mathrm{N}$ & $0.029 \pm 0.011$ & 0.027 & $0.046 \pm 0.013$ & 0.045 & $0.062 \pm 0.022$ & 0.058 & & \\
\hline Maximum stress & $\mathrm{MPa}$ & $0.026 \pm 0.007$ & 0.027 & $0.018 \pm 0.009$ & 0.016 & $0.030 \pm 0.013$ & 0.029 & & \\
\hline Flexural strain & $\%$ & $5.04 \pm 1.00$ & 5.08 & $7.67 \pm 1.95$ & 7.81 & $6.78 \pm 1.64$ & 6.85 & & \\
\hline Max. deflection & $\mathrm{mm}$ & $15.89 \pm 1.71$ & 15.92 & $15.11 \pm 3.45$ & 16.21 & $15.15 \pm 2.96$ & 15.84 & & \\
\hline Sec. m. of area & $\mathrm{mm}^{4}$ & $0.116 \pm 0.122$ & 0.077 & $0.793 \pm 0.498$ & 0.802 & $0.420 \pm 0.291$ & 0.334 & & \\
\hline Flexural rigidity & $\mathrm{N} \cdot \mathrm{mm}^{2}$ & $15.54 \pm 11.09$ & 14.26 & $15.54 \pm 7.44$ & 14.59 & $23.37 \pm 7.69$ & 23.85 & & \\
\hline Flexural modulus & $\mathrm{MPa}$ & $174.56 \pm 88.24$ & 163.37 & $38.50 \pm 41.63$ & 19.69 & $77.41 \pm 47.31$ & 79.08 & & \\
\hline
\end{tabular}


政

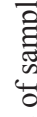

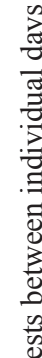

을

䒬

垔

है

竞

苅

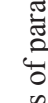

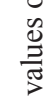

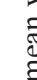

峁

总

这

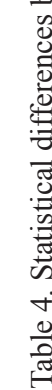

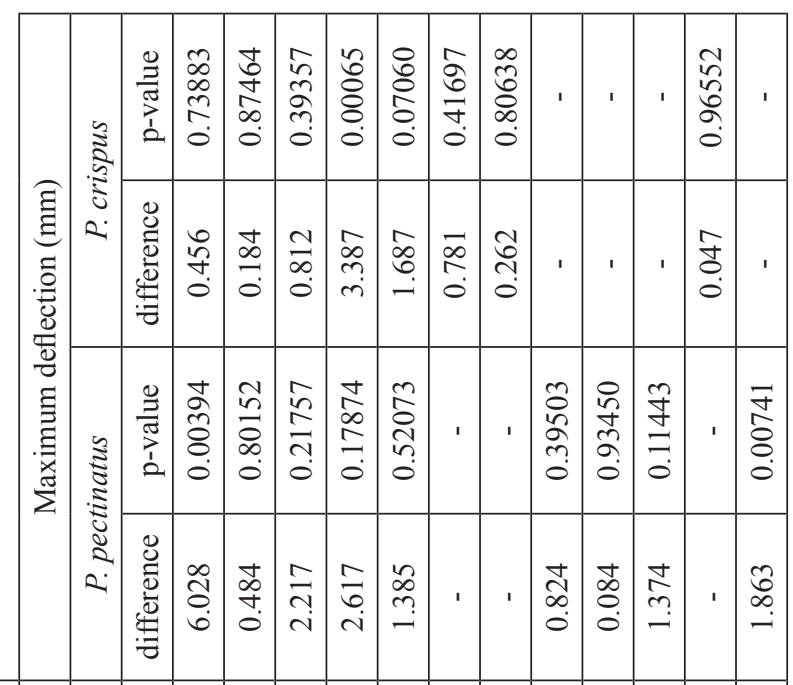

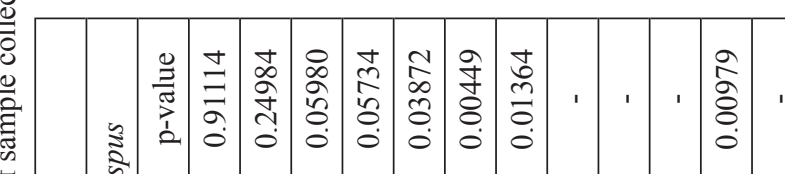

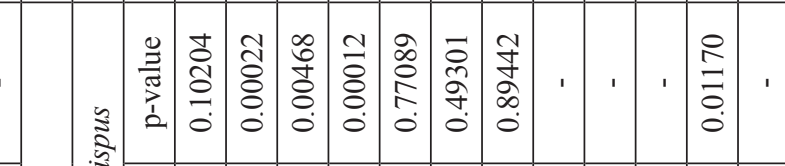

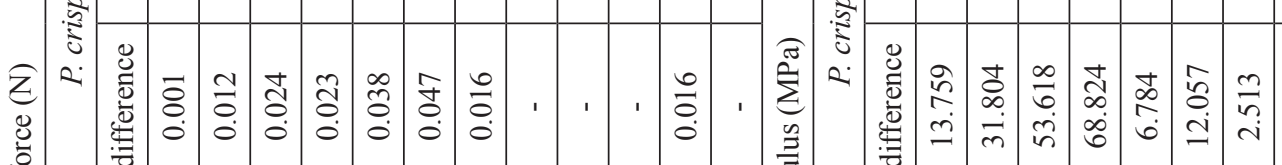

10

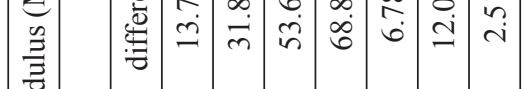

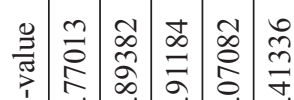

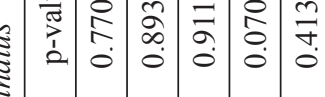

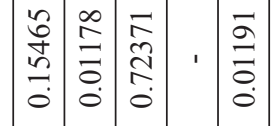

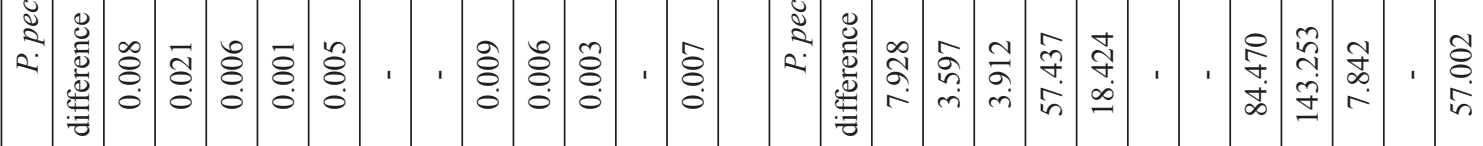

.

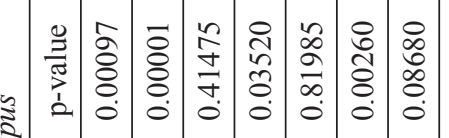

.

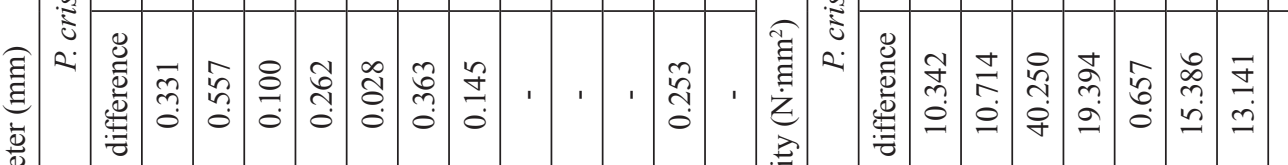

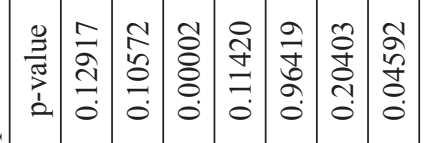

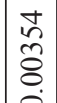

晃

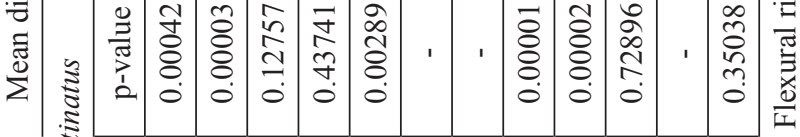

s 5 a

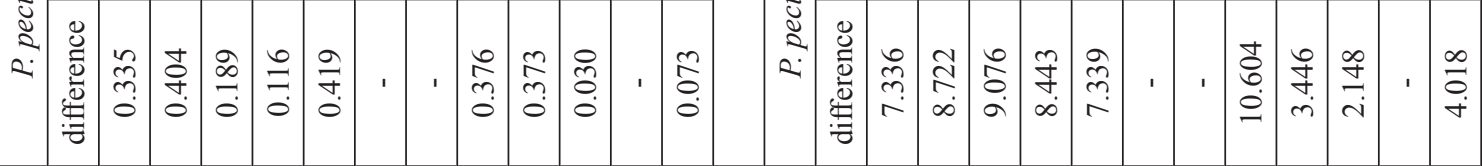

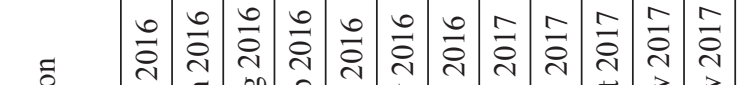

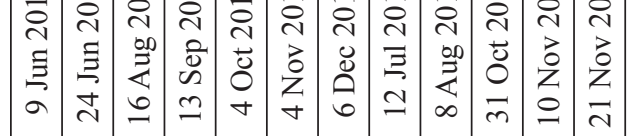

究

$\frac{.5}{\frac{0}{0}}$

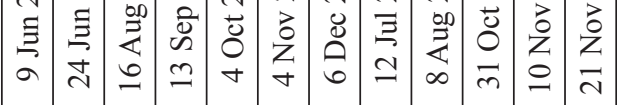

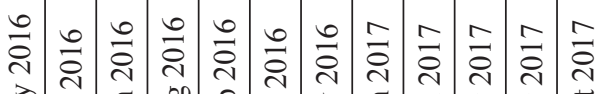

率

突

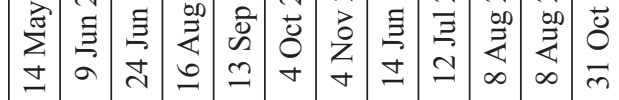

菅

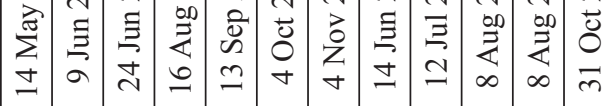


a)
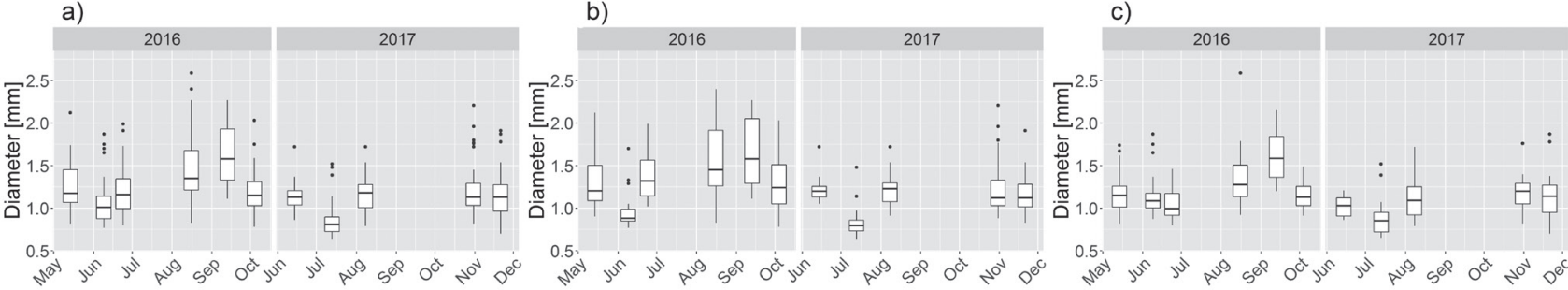

d)

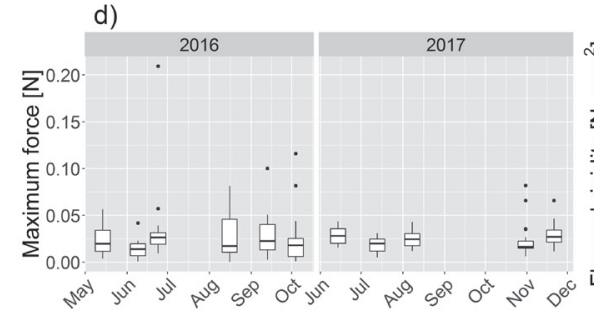

e)
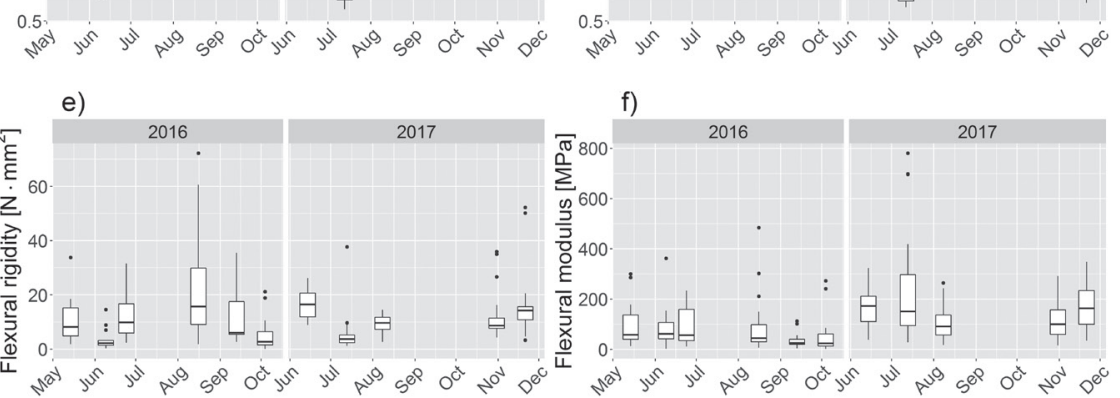

h)
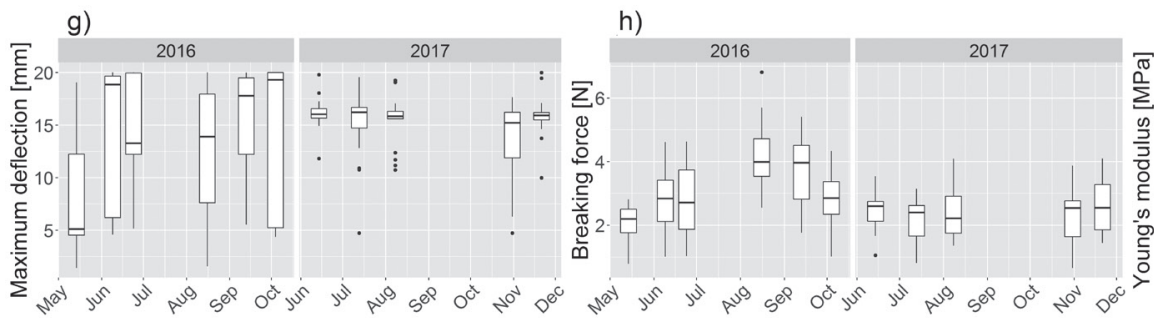

i)

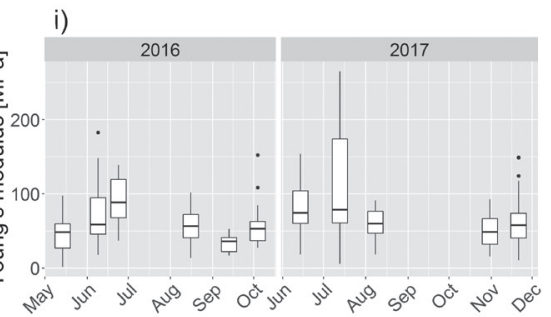

Fig. 7. Diameter of P. pectinatus stems of all specimens from both tests a), specimens from three-point bending tests b), specimens from tension tests c). Biomechanical parameters: maximum force d), flexural rigidity e), flexural modulus f), maximum deflection $g$ ) from three-point bending tests of $P$. pectinatus stems; breaking force $\mathrm{h}$ ), Young's modulus i) from tension tests of plant stems on different days of sample collection in both seasons. The line in the middle of the box is the median, the box edges are the first and third quantiles, the whiskers are minimum and maximum values while the small circles represent outliers.

An increase in discharge was observed again at the beginning of autumn, whereas in winter local flooding is easily recognised. On the other hand, in 2017 the fluctuations in discharge were more frequent and the values were higher (Fig. 4a). During winter and after the spring melt period we observed flooding conditions (Fig. 4a). Later in summer, it stabilised at low values as in the previous year and again the increase began in the autumn (Fig. 4a). In comparison to discharge data, the water temperature was similar in both seasons (Fig. 4b), which was in line with the air temperature (Fig. 4c). In addition, the hydrological and meteorological data, specifically the water and air temperatures, showed that between these two years there was a shift toward warmer temperatures that started in the 2017 growing season (Fig. 4b-c).

\section{Morphology of Plants}

$P$. pectinatus is characterised by thinner stems in comparison to $P$. crispus The samples were chosen randomly, and hence the means of cross-section dimensions differed between both mechanical tests. In 2016 the mean diameter of all $P$. pectinatus specimens in the three-point bending tests ranged from 0.97 to $1.68 \mathrm{~mm}$ in various periods of measurements (Table 2), whereas in 2017 this parameter varied from
0.84 to $1.24 \mathrm{~mm}$ (Table 3 ). The statistically significant differences confirm the decrease in diameter, which can be observed between 14 May and 9 June 2016, and 13 September and 4 October 2016, as well as between 14 June and 12 July 2017 (p-value $<0.05$, Table 4, Fig. 7b). A substantial increase occurred between 9 June and 24 June 2016, and 12 July and 8 August 2017 (p-value $<0.05$, Table 4 , Fig. 7b). In tension tests, these values were between 1.05 and $1.60 \mathrm{~mm}$ in 2016 (Table 6) and between 0.89 and $1.33 \mathrm{~mm}$ in 2017 (Table 7), with significant increases between 24 June and 16 August 2016, 16 August and 13 September 2016, and 12 July and 8 August 2017 (p-value $<0.05$, Table 5, Fig. 7c). The decreases occurred between 13 September and 4 October 2016, and between 14 June and 12 July 2017 (p-value < 0.05, Table 5, Fig. 7c). For P. crispus, the mean values of diameter in the three-point bending tests varied from 1.70 to $2.43 \mathrm{~mm}$ during the first season (Table 2) and from 1.65 to $1.90 \mathrm{~mm}$ during the second season (Table 3). The changes between 14 May and 9 June 2016, and 16 August and 13 September 2016 can be considered statistically significant increases, whereas between 9 June and 24 June 2016, 4 October and 4 November 2016, and 8 August and 10 November 2017 there was a significant decrease ( $p$-value $<0.05$, Table 4 , Fig. $8 \mathrm{~b}$ ). The stem diameters in the tension tests ranged from 1.70 to $2.12 \mathrm{~mm}$ in different sampling periods 


\begin{tabular}{|c|c|c|c|c|c|c|c|c|c|c|c|c|c|c|}
\hline & & 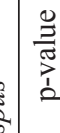 & 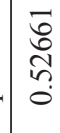 & 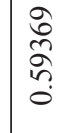 & \begin{tabular}{l}
$\infty$ \\
$\infty$ \\
$\infty$ \\
$\infty$ \\
\hdashline \\
0
\end{tabular} & \begin{tabular}{|l|}
$\stackrel{R}{\hat{~}}$ \\
d. \\
0 \\
0
\end{tabular} & $\mid \begin{array}{c}\tilde{N} \\
\hat{\alpha} \\
\infty \\
\infty \\
0 \\
0\end{array}$ & $\mid \begin{array}{l}\tilde{O} \\
\hat{\Omega} \\
\hat{0}\end{array}$ & $\mid \begin{array}{l}\infty \\
\infty \\
n \\
n \\
n \\
0\end{array}$ & ' & ' & . & 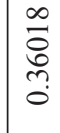 & \\
\hline & $e^{\circ}$ & 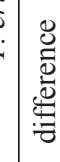 & i & $\begin{array}{l}\infty \\
\infty \\
\sim \\
\sim\end{array}$ & $\begin{array}{l}\text { त̂ } \\
\text { in }\end{array}$ & $\begin{array}{c}\widehat{y} \\
\sigma \\
\sigma\end{array}$ & \begin{tabular}{|l}
0 \\
0 \\
0 \\
0 \\
0
\end{tabular} & $\begin{array}{l}\mathbf{1} \\
\stackrel{\infty}{0} \\
\stackrel{0}{0}\end{array}$ & $\begin{array}{l}\widehat{\infty} \\
\infty \\
\infty \\
-\end{array}$ & ' & ' & ' & $\begin{array}{l}\mathscr{D} \\
\stackrel{\infty}{n}\end{array}$ & \\
\hline & 急 & 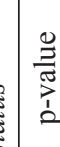 & $\begin{array}{l}8 \\
8 \\
0 \\
0 \\
0\end{array}$ & 站 & 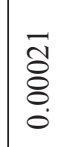 & 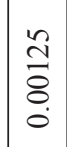 & 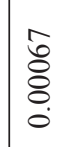 & ' & ' & $\frac{\mathfrak{I}}{\stackrel{0}{I}}$ & $\begin{array}{l}0 \\
0 \\
8 \\
0 \\
0\end{array}$ & 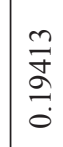 & & 总 \\
\hline & & 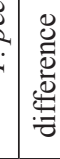 & 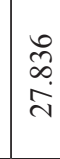 & $\begin{array}{l}\bar{\Xi} \\
\dot{a} \\
\dot{n}\end{array}$ & 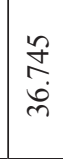 & $\mid \begin{array}{l}\infty \\
\infty \\
\infty \\
\vec{\sim} \\
\end{array}$ & $\begin{array}{l}\vec{b} \\
+ \\
\dot{d}\end{array}$ & ' & ' & $\begin{array}{l}\text { 足 } \\
0 \\
\dot{j} \\
\dot{m}\end{array}$ & $\begin{array}{l}\infty \\
\stackrel{\infty}{0} \\
\stackrel{6}{n}\end{array}$ & 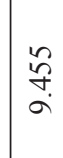 & & $\begin{array}{l}\text { 年 } \\
\\
\end{array}$ \\
\hline & ב & 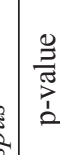 & 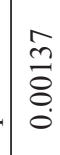 & 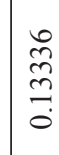 & $\begin{array}{l}\tilde{N} \\
\hat{\sigma} \\
0 \\
0 \\
0\end{array}$ & $\mid \begin{array}{l}\tilde{R} \\
\hat{0} \\
\tilde{0} \\
0 \\
\end{array}$ & 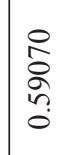 & $\begin{array}{l}\infty \\
\infty \\
\vec{f} \\
\dot{0} \\
0\end{array}$ & $\begin{array}{l}\vec{\sim} \\
\infty \\
0 \\
0 \\
0\end{array}$ & ' & ' & ' & $\begin{array}{l}0 \\
\infty \\
\infty \\
0 \\
0 \\
0 \\
0\end{array}$ & \\
\hline & 2 & 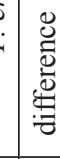 & & $\begin{array}{l}\overline{\widehat{J}} \\
0\end{array}$ & $\begin{array}{l}n \\
0 \\
0\end{array}$ & $\begin{array}{l}t \\
0 \\
0 \\
0 \\
0\end{array}$ & $\begin{array}{l}0 \\
\stackrel{+}{0} \\
0\end{array}$ & 总 & $\stackrel{ }{I}$ & ' & ' & ' & 守 & \\
\hline & 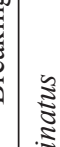 & 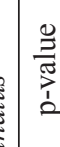 & $\mid \begin{array}{l}\tilde{n} \\
0 \\
0 \\
0\end{array}$ & 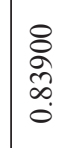 & 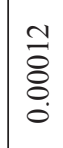 & 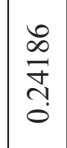 & 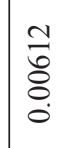 & ' & ' & 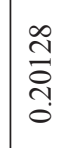 & $\begin{array}{l}\overline{\widetilde{O}} \\
\text { ఫे. }\end{array}$ & 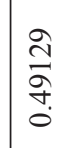 & 1 & $\frac{\bar{\infty}}{\bar{\alpha}}$ \\
\hline & 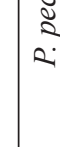 & 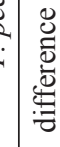 & 옹 & 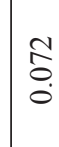 & $\underset{f}{\stackrel{f}{f}}$ & 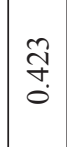 & $\begin{array}{l}\bar{n} \\
\hat{o}\end{array}$ & ' & ' & 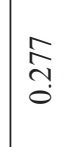 & $\stackrel{\overline{\hat{~}}}{0}$ & $\begin{array}{l}\text { ָิ } \\
\text { ô }\end{array}$ & & 皿 \\
\hline & בె & 20 & 立 & 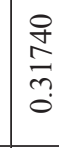 & $\mid \begin{array}{l}0 \\
0 \\
0 \\
0 \\
0 \\
0\end{array}$ & 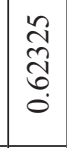 & $\mid \begin{array}{l}m \\
\vec{a} \\
\vdots \\
0 \\
0\end{array}$ & 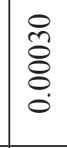 & 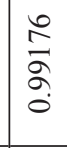 & ' & ' & ' & $\begin{array}{l}2 \\
\tilde{O} \\
0 \\
0 \\
0\end{array}$ & \\
\hline & 2 & 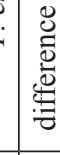 & 令 & 守 & $\begin{array}{c}\vec{J} \\
\tilde{O} \\
\end{array}$ & $\begin{array}{l}+ \\
\stackrel{H}{0} \\
0 \\
0\end{array}$ & $\begin{array}{l}0 \\
0 \\
0 \\
0\end{array}$ & 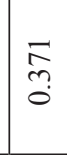 & 官 & ' & I & ' & \begin{tabular}{|l}
$\infty$ \\
0 \\
1 \\
0
\end{tabular} & \\
\hline & 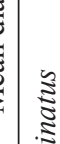 & 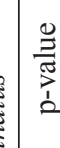 & 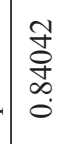 & $\begin{array}{c}\tilde{b} \\
\stackrel{0}{0} \\
0\end{array}$ & $\mid \begin{array}{l}m \\
0 \\
8 \\
0 \\
0 \\
0\end{array}$ & $\mid$\begin{tabular}{l|}
$\overrightarrow{0}$ \\
$\dot{0}$ \\
0 \\
0 \\
0
\end{tabular} & $\begin{array}{l}\bar{\delta} \\
\dot{\delta} \\
\dot{0}\end{array}$ & ' & ' & \begin{tabular}{l}
0 \\
$\infty$ \\
\multirow{2}{*}{} \\
0 \\
0
\end{tabular} & $\begin{array}{l}n \\
\hat{n} \\
\stackrel{n}{0} \\
0 \\
0\end{array}$ & $\begin{array}{l}\tilde{y} \\
\text { ơ } \\
\text { co } \\
0\end{array}$ & & 号 \\
\hline & §. & 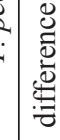 & $\stackrel{\infty}{\stackrel{\infty}{\circ}}$ & $\stackrel{2}{a}$ & $\begin{array}{l}n \\
\tilde{o} \\
0\end{array}$ & $\stackrel{\check{T}}{\tilde{\sigma}}$ & 导 & ' & ' & సे & तี & : & & $\tilde{\tilde{o}}$ \\
\hline & 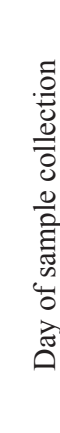 & & 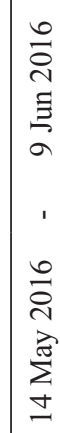 & 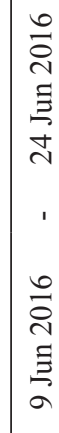 & 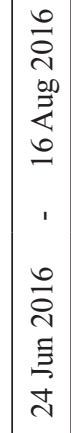 & 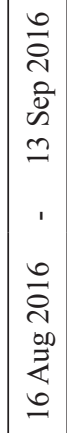 & 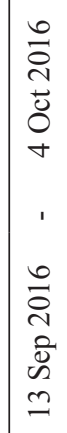 & 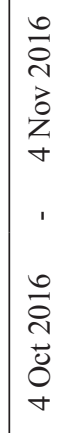 & 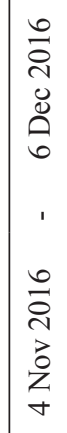 & 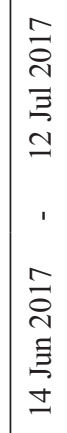 & 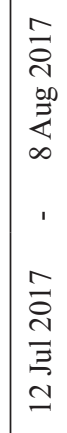 & 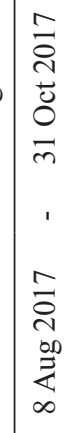 & $\begin{array}{l}\text { ㄱ. } \\
\text { N } \\
\text { zे } \\
z \\
0 \\
- \\
.\end{array}$ & $\mid \begin{array}{l}n \\
\bar{C} \\
z \\
z \\
z\end{array}$ \\
\hline
\end{tabular}


Table 6. Diameter and tension test results for all periods of measurement of P. pectinatus L. and P. crispus L. in 2016.

\begin{tabular}{|c|c|c|c|c|c|c|c|c|c|}
\hline \multicolumn{10}{|c|}{ P. pectinatus } \\
\hline \multirow{2}{*}{\multicolumn{2}{|c|}{ Parameter }} & \multicolumn{2}{|c|}{14 May } & \multicolumn{2}{|c|}{9 June } & \multicolumn{2}{|c|}{24 June } & \multicolumn{2}{|c|}{16 Aug. } \\
\hline & & Mean \pm S.D. & Median & Mean \pm S.D. & Median & Mean \pm S.D. & Median & Mean \pm S.D. & Median \\
\hline \multicolumn{2}{|c|}{ Number of samples } & \multicolumn{2}{|l|}{20} & \multicolumn{2}{|l|}{20} & \multicolumn{2}{|c|}{20} & \multicolumn{2}{|c|}{20} \\
\hline Diameter & $\mathrm{mm}$ & $1.19 \pm 0.25$ & 1.15 & $1.17 \pm 0.28$ & 1.09 & $1.05 \pm 0.19$ & 1.00 & $1.38 \pm 0.37$ & 1.28 \\
\hline Breaking force & $\mathrm{N}$ & $2.05 \pm 0.59$ & 2.20 & $2.82 \pm 1.15$ & 2.84 & $2.75 \pm 1.09$ & 2.71 & $4.19 \pm 1.03$ & 3.99 \\
\hline Breaking stress & $\mathrm{MPa}$ & $2.07 \pm 0.93$ & 2.17 & $2.81 \pm 1.34$ & 2.66 & $3.30 \pm 1.25$ & 3.48 & $3.01 \pm 0.87$ & 3.25 \\
\hline Breaking strain & $\%$ & $7.04 \pm 1.88$ & 6.52 & $7.48 \pm 2.29$ & 7.84 & $6.22 \pm 2.10$ & 5.65 & $11.13 \pm 2.30$ & 11.34 \\
\hline Young's modulus & $\mathrm{MPa}$ & $47.79 \pm 26.58$ & 48.42 & $75.62 \pm 44.33$ & 58.51 & $91.52 \pm 32.25$ & 88.25 & $54.78 \pm 23.61$ & 56.48 \\
\hline \multirow{2}{*}{\multicolumn{2}{|c|}{$\begin{array}{c}\text { Parameter } \\
\text { Mean } \pm \text { S.D. }\end{array}$}} & \multicolumn{2}{|c|}{13 Sept. } & \multicolumn{2}{|c|}{4 Oct. } & \multicolumn{2}{|c|}{4 Nov. } & \multicolumn{2}{|c|}{6 Dec. } \\
\hline & & Mean \pm S.D. & Median & Mean \pm S.D. & Median & Mean \pm S.D. & Median & Mean \pm S.D. & Median \\
\hline \multicolumn{2}{|c|}{ Number of samples } & \multicolumn{2}{|l|}{18} & \multicolumn{2}{|l|}{20} & \multicolumn{2}{|l|}{-} & \multicolumn{2}{|l|}{-} \\
\hline Diameter & $\mathrm{mm}$ & $1.60 \pm 0.28$ & 1.59 & $1.15 \pm 0.16$ & 1.13 & - & - & - & - \\
\hline Breaking force & $\mathrm{N}$ & $3.77 \pm 1.17$ & 3.96 & $2.81 \pm 0.80$ & 2.86 & - & - & - & - \\
\hline Breaking stress & $\mathrm{MPa}$ & $1.86 \pm 0.36$ & 1.85 & $2.75 \pm 0.82$ & 2.68 & - & - & - & - \\
\hline Breaking strain & $\%$ & $10.31 \pm 1.82$ & 10.38 & $8.05 \pm 1.48$ & 8.11 & - & - & - & - \\
\hline Young's modulus & $\mathrm{MPa}$ & $32.92 \pm 11.60$ & 36.05 & $57.38 \pm 30.23$ & 52.83 & - & - & - & - \\
\hline \multicolumn{10}{|c|}{ P. crispus } \\
\hline \multirow{2}{*}{\multicolumn{2}{|c|}{$\begin{array}{c}\text { Parameter } \\
\text { Mean } \pm \text { S.D. }\end{array}$}} & \multicolumn{2}{|c|}{14 May } & \multicolumn{2}{|c|}{9 June } & \multicolumn{2}{|c|}{24 June } & \multicolumn{2}{|c|}{16 Aug. } \\
\hline & & Mean \pm S.D. & Median & Mean \pm S.D. & Median & Mean \pm S.D. & Median & Mean \pm S.D. & Median \\
\hline Number of samp & oles & 20 & & 19 & & 19 & & 19 & \\
\hline Diameter & $\mathrm{mm}$ & $2.01 \pm 0.30$ & 2.04 & $2.08 \pm 0.40$ & 2.09 & $1.94 \pm 0.45$ & 1.78 & $1.70 \pm 0.38$ & 1.77 \\
\hline Breaking force & $\mathrm{N}$ & $2.44 \pm 0.84$ & 2.26 & $3.84 \pm 1.58$ & 3.66 & $3.22 \pm 0.76$ & 3.21 & $3.11 \pm 0.87$ & 3.18 \\
\hline Breaking stress & $\mathrm{MPa}$ & $0.80 \pm 0.31$ & 0.70 & $1.17 \pm 0.52$ & 1.03 & $1.20 \pm 0.47$ & 1.08 & $1.61 \pm 0.92$ & 1.37 \\
\hline Breaking strain & $\%$ & $7.77 \pm 3.27$ & 7.66 & $6.68 \pm 1.89$ & 5.94 & $7.79 \pm 2.48$ & 7.61 & $7.73 \pm 3.14$ & 7.25 \\
\hline Young's modulus & $\mathrm{MPa}$ & $16.34 \pm 7.72$ & 15.26 & $18.98 \pm 15.63$ & 12.98 & $21.37 \pm 10.60$ & 19.08 & $26.60 \pm 13.35$ & 25.59 \\
\hline Parameter & & $13 \mathrm{Se}$ & & $4 \mathrm{Oc}$ & & $4 \mathrm{No}$ & & $6 \mathrm{De}$ & \\
\hline Mean \pm S.D. & & Mean \pm S.D. & Median & Mean \pm S.D. & Median & Mean \pm S.D. & Median & Mean \pm S.D. & Median \\
\hline Number of samp & oles & 17 & & 22 & & 19 & & 20 & \\
\hline Diameter & $\mathrm{mm}$ & $1.76 \pm 0.26$ & 1.77 & $2.12 \pm 0.27$ & 2.03 & $1.75 \pm 0.34$ & 1.78 & $1.74 \pm 0.28$ & 1.70 \\
\hline Breaking force & $\mathrm{N}$ & $3.68 \pm 1.96$ & 3.15 & $4.17 \pm 3.28$ & 2.99 & $2.57 \pm 0.64$ & 2.53 & $2.40 \pm 1.46$ & 1.74 \\
\hline Breaking stress & $\mathrm{MPa}$ & $1.46 \pm 0.55$ & 1.29 & $1.12 \pm 0.74$ & 0.91 & $1.17 \pm 0.49$ & 0.98 & $1.03 \pm 0.55$ & 0.89 \\
\hline Breaking strain & $\%$ & $8.97 \pm 2.56$ & 8.06 & $7.42 \pm 2.70$ & 7.75 & $8.39 \pm 2.50$ & 8.72 & $6.85 \pm 2.65$ & 6.04 \\
\hline Young's modulus & $\mathrm{MPa}$ & $17.18 \pm 10.91$ & 15.59 & $17.64 \pm 8.34$ & 16.84 & $16.86 \pm 10.40$ & 14.11 & $18.75 \pm 9.48$ & 16.97 \\
\hline
\end{tabular}

in 2016 (Table 6), while in 2017 the values obtained were between 1.44 and $1.70 \mathrm{~mm}$ (Table 7). The calculations show that between 13 September and 4 October 2016 there was a significant increase, while a substantial decrease was observed between 4 October and 4 November 2016, and 8 August and 10 November 2017 (p-value $<0.05$, Table 5, Fig. 8c).

The two species tested from the family Potamogetonaceae differ in structure (Figs 5 and 6).
The cross-sectional area of $P$. pectinatus has a circular shape, while in P. crispus, the form resembles an ellipse (Fig. 6). However, both species have a honeycomb aerenchyma structure (Fig. 6). In the first season in 2016, P. pectinatus was most developed in September, but the plant was characterised by a lower number of leaves (Fig. 5). Additionally, the time when P. crispus was in full bloom is difficult to estimate due to its unique life cycle [20] (Fig. 5). 


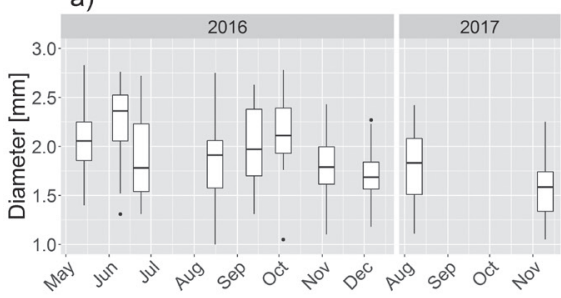

d)

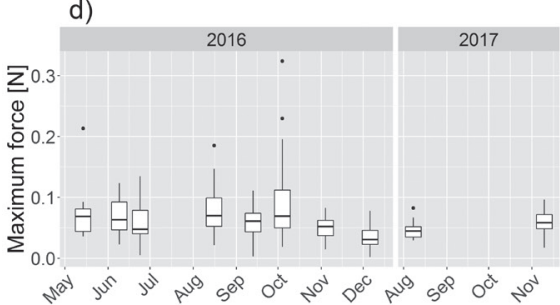

g)

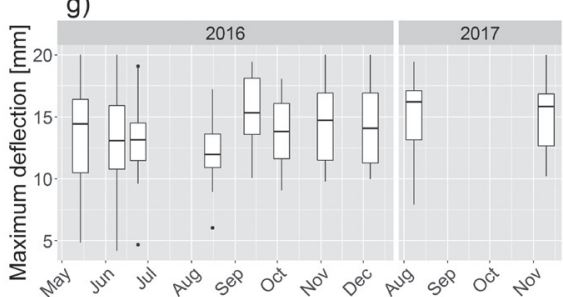

b)

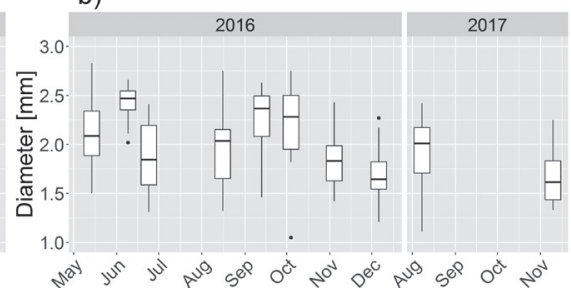

e)

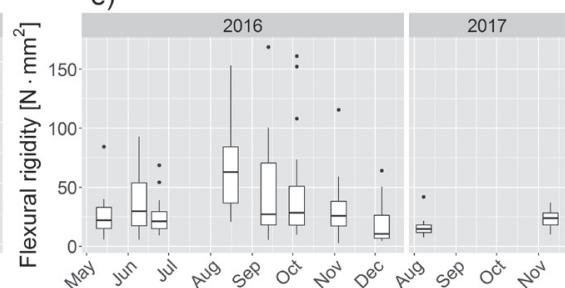

h)

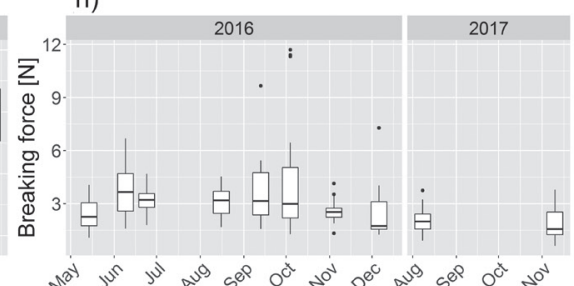

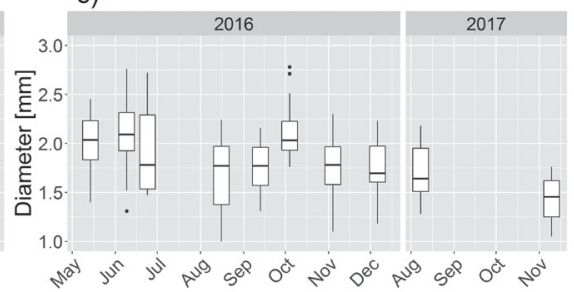

f)

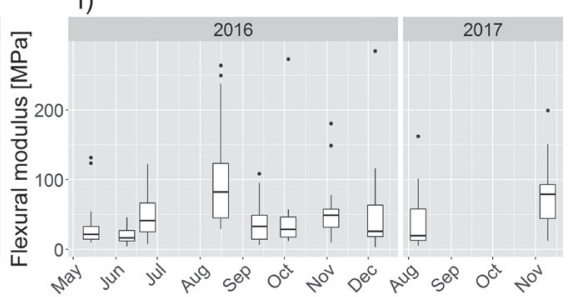

i)

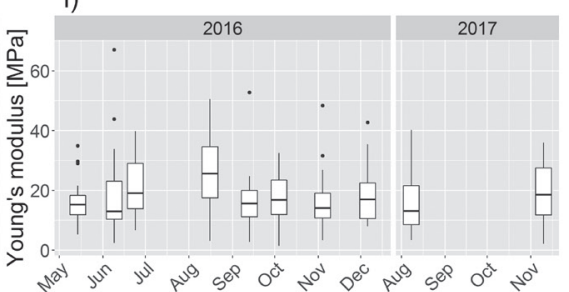

Fig. 8. Diameter of P. crispus stems of all specimens from both tests a), specimens from three-point bending tests b), specimens from tension tests c). Biomechanical parameters: maximum force d), flexural rigidity e), flexural modulus f), maximum deflection $g$ ) from three-point bending tests of $P$. crispus stems; breaking force h), Young's modulus i) from tension tests of plant stems on different days of sample collection in both seasons. The line in the middle of the box is the median, the box edges are the first and third quantiles, the whiskers are minimum and maximum values while the small circles represent outliers.

Table 7. Diameter and tension test results for all periods of measurements of P. pectinatus and P. crispus in 2017.

\begin{tabular}{|c|c|c|c|c|c|c|c|c|c|}
\hline \multicolumn{10}{|c|}{ P. pectinatus } \\
\hline \multirow{2}{*}{\multicolumn{2}{|c|}{ Parameter }} & \multicolumn{2}{|c|}{14 June } & \multicolumn{2}{|c|}{12 July } & \multicolumn{2}{|c|}{8 Aug. } & \multicolumn{2}{|c|}{31 Oct. } \\
\hline & & Mean \pm S.D. & Median & Mean \pm S.D. & Median & Mean \pm S.D. & Median & Mean \pm S.D. & Median \\
\hline \multicolumn{2}{|c|}{ Number of samples } & \multicolumn{2}{|l|}{19} & \multicolumn{2}{|l|}{17} & \multicolumn{2}{|l|}{19} & \multicolumn{2}{|c|}{25} \\
\hline Diameter & $\mathrm{mm}$ & $1.02 \pm 0.12$ & 1.03 & $0.89 \pm 0.24$ & 0.85 & $1.10 \pm 0.24$ & 1.09 & $1.17 \pm 0.19$ & 1.20 \\
\hline Breaking force & $\mathrm{N}$ & $2.47 \pm 0.60$ & 2.60 & $2.19 \pm 0.68$ & 2.40 & $2.41 \pm 0.88$ & 2.22 & $2.20 \pm 0.94$ & 2.54 \\
\hline Breaking stress & $\mathrm{MPa}$ & $3.06 \pm 0.87$ & 2.93 & $4.12 \pm 2.24$ & 3.82 & $2.52 \pm 0.50$ & 2.68 & $2.08 \pm 0.81$ & 2.23 \\
\hline Breaking strain & $\%$ & $7.04 \pm 1.99$ & 7.71 & $7.93 \pm 2.87$ & 7.88 & $9.21 \pm 2.65$ & 9.40 & $7.23 \pm 2.39$ & 7.92 \\
\hline Young's modulus & $\mathrm{MPa}$ & $82.18 \pm 38.57$ & 74.33 & $116.23 \pm 83.72$ & 78.71 & $60.05 \pm 21.61$ & 59.76 & $50.60 \pm 23.86$ & 48.52 \\
\hline & & \multicolumn{2}{|c|}{ P. pectinatus } & \multicolumn{4}{|c|}{ P. crispus } & & \\
\hline \multirow{2}{*}{\multicolumn{2}{|c|}{$\begin{array}{c}\text { Parameter } \\
\text { Mean } \pm \text { S.D. }\end{array}$}} & \multicolumn{2}{|c|}{21 Nov. } & \multicolumn{2}{|c|}{8 Aug. } & \multicolumn{2}{|c|}{10 Nov. } & & \\
\hline & & Mean \pm S.D. & Median & Mean \pm S.D. & Median & Mean \pm S.D. & Median & & \\
\hline \multicolumn{2}{|c|}{ Number of samples } & \multicolumn{2}{|l|}{27} & \multicolumn{2}{|l|}{19} & \multicolumn{2}{|l|}{12} & & \\
\hline Diameter & $\mathrm{mm}$ & $1.33 \pm 0.27$ & 1.13 & $1.70 \pm 0.28$ & 1.64 & $1.44 \pm 0.24$ & 1.46 & & \\
\hline Breaking force & $\mathrm{N}$ & $2.62 \pm 0.79$ & 2.55 & $2.06 \pm 0.72$ & 1.99 & $1.91 \pm 1.07$ & 1.56 & & \\
\hline Breaking stress & $\mathrm{MPa}$ & $2.72 \pm 0.82$ & 2.60 & $0.95 \pm 0.44$ & 0.80 & $1.27 \pm 0.83$ & 0.99 & & \\
\hline Breaking strain & $\%$ & $8.52 \pm 2.38$ & 8.79 & $7.02 \pm 2.61$ & 6.65 & $6.65 \pm 2.30$ & 6.90 & & \\
\hline Young's modulus & $\mathrm{MPa}$ & $64.14 \pm 36.56$ & 57.83 & $15.75 \pm 9.37$ & 13.13 & $19.13 \pm 10.59$ & 18.57 & & \\
\hline
\end{tabular}




\section{Comparison between Seasons}

The differences in diameters from three-point bending and tension tests, flexural rigidity, and Young's modulus between similar measurement periods of the first and second seasons were investigated. The results for the three-point bending tests of $P$. pectinatus showed that there were significant differences in diameter between 9 June 2016 and 14 June 2017, 24 June 2016 and 12 July 2017, and 16 August 2016 and 8 August 2017 (p-value $<0.05$, Table 8). As for flexural rigidity, significant changes were observed in all investigated periods ( $p$-value $<0.05$, Table 8). In comparison to the three-point bending tests, in tension tests the only significant difference occurred in diameter between 16 August 2016 and 8 August 2017 ( $p$-value $<0.05$, Table 8 ). Young's modulus values did not change significantly (Table 8). For P. crispus, despite no changes in diameter between 16 August 2016 and 8 August 2017 in both tests ( $p$-value $<0.05$, Table 8 ), the comparison showed significant differences in biomechanical parameters, i.e., flexural rigidity and Young's modulus ( $p$-value $<0.05$, Table 8). On the other hand, the statistical analysis of these parameters between 4 November 2016 and 10 November 2017 showed significant differences in diameters ( $p$-value $<0.05$, Table 8); however, for flexural rigidity and Young's modulus, there were no changes.

\section{Three-Point Bending Tests}

In 2016, at the beginning of the investigated period between 14 May and 9 June, the maximum force and the flexural rigidity of $P$. pectinatus significantly decreased ( $p$-value $<0.05$, Table 4, Fig. 7d-e). Then, those values rose rapidly between 9 June and 24 June, which was confirmed by statistical analysis (p-value $<0.05$, Tables 2 and 4, Fig. 7d-e). From the end of June to October, the variations in force outcomes were not statistically significant. Despite this phenomenon, changes in the mechanics of $P$. pectinatus were observed. The significant drop in the flexural rigidity in October ( $p$-value $<0.05$, Table 4, Fig. 7e) occurred after a period when the plant was characterised by the highest stiffness, with mean values in August and September equalling $21.09 \mathrm{~N} \cdot \mathrm{mm}^{2}$ and $12.65 \mathrm{~N} \cdot \mathrm{mm}^{2}$, respectively (Table 2). The statistical analysis has shown that there were no significant changes in the flexural modulus between the investigated periods. However, there was a visible downward trend with passing time (Table 2, Fig. 7f). During the next season in 2017, the statistical analysis showed a decrease in maximum force and flexural rigidity between 14 June and 12 July ( $p$-value $<0.05$, Table 4, Fig. $7 d-e$ ). Then, the maximum force significantly increased between 12 July and 8 August (p-value $<0.05$, Table 4 , Fig. $7 d$ ). At the same time, there was observed drop in the flexural modulus, while an increase occurred between 31 October and 21 November (p-value $<0.05$, Table 4, Fig. 7f). Significant changes in maximum deflection were observed between October 31 and November 21 ( $p$-value $<0.05$, Table 4, Fig. $7 g$ ).

On the other hand, in $2016 P$. crispus was characterised by a substantially increasing flexural modulus that mimicked plant growth. The noticeable increase in this parameter was observed between 9 June and 24 June, and 24 June and 16 August (p-value $<0.05$, Table 4, Fig. 8f), when it reached a mean value of 105.21 MPa (Table 2). After this period, the modulus of elasticity significantly decreased to 36.38 MPa (p-value $<0.05$, Tables 2 and 4, Fig. 8f). In contrast to $P$. pectinatus, the maximum bending force of $P$. crispus. was balanced during the warmer months (May-September) and subsequently increased in October (p-value $<0.05$, Tables 2 and 4, Fig. 8d). After that, a significant decrease in bending strength was observed to the end of the investigated period ( $\mathrm{p}$-value $<0.05$, Table 4, Fig. 8d). For flexural rigidity, statistically significant changes ( $p$-value $<0.05$, Table 2, Fig. 8 e) were obtained between 24 June and 16 August, and between 4 November and 6 December. Like P. pectinatus, the highest mean value was observed in August, equalling $65.67 \mathrm{~N} \cdot \mathrm{mm}^{2}$ (Table 2). In the second season, this species was found in exactly the same place twice, and statistical calculations between periods (8 August and 10 November) showed significant increases in maximum force, flexural rigidity, and flexural modulus (p-value $<0.05$, Table 4, Fig. 8d-f).

In a comparison of both hydrophyte species, differences in outcomes of the three-point bending parameters were visible. The forces needed to completely fracture $P$. crispus were much higher than those needed to fracture $P$. pectinatus A direct reason could be the greater stiffness resulting from the thickness of shoots, which was two to ten times greater than in $P$. crispus (Table 2). In contrast to flexural rigidity, a larger resistance to being deformed elastically was obtained for $P$. pectinatus due to its thinner shoots (Table 2).

\section{Tension Tests}

During the first investigated season in 2016, statistically significant variations in mean values of breaking forces for $P$. pectinatus $\mathrm{L}$. were observed between 14 May and 9 June, 24 June and 16 August, and 13 September and 4 October ( $p$-value $<0.05$, Table 5, Fig. 7h). There was an increase in plant strength until August, with a maximum value of $4.19 \mathrm{~N}$ (Table 6), whereas at the end of the vegetative season there was visible drop in values to $2.81 \mathrm{~N}$ on the 4 October (Table 6). In the period between May and June, the shoot dimensions were similar, and no significant changes were noted ( $p$-value $<0.05$, Table 5, Fig. 7c). Nevertheless, Young's modulus increased sharply from 47.79 up to a maximum value of $91.52 \mathrm{MPa}$ (Table 6). Furthermore, even with increasing plant dimensions, shoot elasticity drastically decreased to $32.92 \mathrm{MPa}$ 
Table 8. Statistical differences in the mean values of the diameter and flexural rigidity from the three-point bending test; the diameter and Young's modulus from the tension tests between similar measurement periods from the first and second seasons for $P$. pectinatus and P. crispus.

\begin{tabular}{|c|c|c|c|c|c|c|c|c|c|c|}
\hline \multirow{3}{*}{\multicolumn{3}{|c|}{ Day of sample collection }} & \multicolumn{4}{|c|}{ Three-point bending test } & \multicolumn{4}{|c|}{ Tension test } \\
\hline & & & \multicolumn{2}{|c|}{$\begin{array}{l}\text { Diameter } \\
(\mathrm{mm})\end{array}$} & \multicolumn{2}{|c|}{$\begin{array}{l}\text { Flexural rigidity } \\
\left(\mathrm{N} \cdot \mathrm{mm}^{2}\right)\end{array}$} & \multicolumn{2}{|c|}{$\begin{array}{c}\text { Diameter } \\
(\mathrm{mm})\end{array}$} & \multicolumn{2}{|c|}{$\begin{array}{l}\text { Young's modulus } \\
(\mathrm{MPa})\end{array}$} \\
\hline & & & difference & p-value & difference & $\mathrm{p}$-value & difference & p-value & difference & $\mathrm{p}$-value \\
\hline \multicolumn{11}{|c|}{ P. pectinatus } \\
\hline 9 Jun 2016 & & 14 Jun 2017 & 0.25 & 0.00023 & 13.240 & 0.00001 & 0.153 & 0.02844 & 6.554 & 0.62476 \\
\hline 24 Jun 2016 & - & 12 Jul 2017 & 0.532 & 0.00001 & 6.086 & 0.02746 & 0.157 & 0.03287 & 24.710 & 0.23322 \\
\hline 16 Aug 2016 & - & 8 Aug 2017 & 0.348 & 0.00308 & 11.716 & 0.00592 & 0.281 & 0.00723 & 5.277 & 0.48248 \\
\hline 4 Oct 2016 & - & 31 Oct 2017 & 0.015 & 0.88269 & 6.214 & 0.00333 & 0.017 & 0.75732 & 6.781 & 0.41406 \\
\hline \multicolumn{11}{|c|}{ P. crispus } \\
\hline 16 Aug 2016 & - & 8 Aug 2017 & 0.068 & 0.59424 & 50.127 & 0.00001 & 0.068 & 0.59424 & 50.127 & 0.00001 \\
\hline 4 Nov 2016 & - & 10 Nov 2017 & 0.191 & 0.04179 & 8.173 & 0.22460 & 0.311 & 0.01147 & 2.275 & 0.56375 \\
\hline
\end{tabular}

(p-value 0.05, Tables 5-6, Fig. 7i). An increase in Young's modulus was observed between September 13 and October 4 (p-value 0.05, Table 5, Fig. 7i). In the second season of 2017, the breaking force of $P$. pectinatus did not change significantly (Table 5); however, there a statistically significant decrease in Young's modulus was observed between 12 July and 8 August (p-value 0.05, Table 5, Fig. 7i). Generally, in various phases of plant growth the results confirm that the biomechanical properties of stems vary according to seasonal changes.

In contrast to $P$. pectinatus, $P$. crispus had more stable properties. An increase in mean breaking force values during the first season was observed between 14 May and 9 June (p-value 0.05, Tables 5-6, Fig. 8h), when the forces increased from 2.44 to $3.84 \mathrm{~N}$ (Table 6). During the summer period, the fluctuations were registered as irrelevant. However, when the water temperature drastically dropped (to $6^{\circ} \mathrm{C}$ on the 4 November, Table 1), a statistically significant decrease in tensile load of approximately $40 \%$ (p-value 0.05 , Tables 5-6, Fig. $8 \mathrm{~h}$ ) was observed. The parameter of elasticity of the plant stem, Young's modulus, remained at a similar level until August. Then, the ability to elastically deform was reduced to $17.18 \mathrm{MPa}$ ( $\mathrm{p}$-value 0.05 , Tables 5-6, Fig. 8i), and it oscillated within $18 \mathrm{MPa}$ (Table 6). In the second season, there were no significant changes in biomechanical properties for this species (Table 5).

\section{Discussion}

The biomechanical behaviour of plants is strongly related to flow-vegetation-sediment interactions [30], which is important for engineering and flood protection. The presented data and calculations based on the biomechanical tests, i.e., three-point bending and tension, showed that fluctuations in mechanical traits occurred in both tested plant species during the investigated seasons. The variations may be a consequence of many factors, e.g., plant growth stage or hydrological and meteorological conditions.

As previously mentioned, Potamogeton spp. were only investigated biomechanically by Brewer and Parker [22], Bociąg et al. [23], and Miler et al. [16]. For instance, $P$. pectinatus was examined for the first time by Brewer and Parker [22]. The plant was subjected to tensile tests; however, the values for tensile strength $(33.8 \mathrm{MPa})$ differed significantly from those we have obtained in all periods of our study (0.80-3.30 MPa, Table 6). Bociag et al. [23] collected individual plants from stagnant and flowing water in July. They found values of breaking force $(3.3 \mathrm{~N})$ for plants coming from rivers that were more comparable to our outcomes around the same time of the year (Table 6). The second species, P. crispus, was investigated by Miler et al. [16]. They obtained lower values of biomechanical traits, i.e., breaking force, Young's modulus, than our results, which may be related to a smaller sample diameter. However, the flexural modulus was much higher than the results presented in this paper. Nevertheless, the comprehensive research on the seasonal variability of the biomechanical properties of these two species is presented for the first time here. The comparison of two seasons for $P$. pectinatus showed that there was a repeating cycle of changes in biomechanical traits (Fig. 7); however, some shifts of similar trends in characteristics have been noticed, i.e., the statistical analysis demonstrated that at the same time of year, the differences in flexural rigidity and diameters from both tests were significant (Table 8, Fig. 7). This shift may be related to changes in hydrological and meteorological conditions. The vegetation season started later in 2017 than in 2016, and it was shorter (Fig. 4b-c). We speculate that this shift is related to a clearly longer period of lower air and water temperatures (Table 1). This phenomenon indicates the need to take a broader perspective in determining 
the biomechanical parameters of a plant from a particular period, e.g., by considering air and water temperatures. According to O'Hare [12], aquatic macrophytes may shift their adjustments to the conditions, and they may become more stress-tolerant. This is confirmed by the differences in the results of the three-point bending test between seasons, where in 2017 plants were characterised by lower flexural rigidity connected with higher flexural modulus, which was responsible for elastic deformation, meaning the return of the plant to its original shape after the load was removed. The higher discharges and water velocities could cause the adoption of an avoidance strategy to prevent damage or destruction, which was characterised by an increase in shoot elasticity in line with the results of the study by Robionek et al. [31].

According to Schutten et al. [32] and Puijalon et al. [33], aquatic plants are flexible and adjust to living factors by bending with the flow direction. This is an example of an avoidance strategy that helps them survive in different hydrological conditions. This statement is confirmed by our results as the Potamogeton spp. requires a higher tensile strength to avoid destruction due to the flexibility of its shoots, which bend easily (Tables 2-3, 6-7). However, the investigated hydrophytes, which have different structures and life cycles, show significant differences in biomechanical properties. Moreover, the flow velocities in the cross-section at the location of the plant habitats differed for both species. $P$. pectinatus grew in a place where the flow was considerably lower than in the habitat of P. crispus (Fig. 1), which suggests that higher resistance of P. crispus is one of the traits of the avoidance strategy adapted by this plant.

\section{Conclusions}

Our results supplement an aquatic plant biomechanics database that has been successfully built during recent years [15-16, 22-23, 32-33], complementing it by providing data on the seasonal changes of two commonly growing aquatic plants, which were, for the first time, collected during two vegetation seasons. We believe that the continuous observation and measurement of biomechanical properties of aquatic plants are required, as they will provide essential information about plant adaptive capacity to changing conditions. This is significant, since hydraulic resistance is the most important factor for ecological and civil engineering management.

Moreover, the following five points summarize our conclusions:

1. The calculations of biomechanical traits - particularly strain, stiffness, and the moduli of elasticity clearly indicate the significant difference in biomechanical properties of two submerged aquatic plants $P$. pectinatus and $P$. crispus at the same moment in phenological time.
2. Potamogetonaceae plants were characterised by approximately similar strains under tension. The main difference was noticeable in the modulus of elasticity values, where $P$. pectinatus was much more prone to return to its original shape after the removal of the acting forces.

3. The differences in the structures and life cycles of the investigated species may be crucial factors that differentiate the mechanical strength to withstand bending and tensile loads of both macrophytes.

4. The hydrological and meteorological conditions may be considered the main factors regulating the values of biomechanical traits of Potamogeton spp., with flexural rigidity being the most sensitive parameter for changes in hydrological conditions.

5. The comparison of data of the biomechanical tests, even for the same species and at the same time of year, may lead to misinterpretation of the obtained results, as the statistical analysis demonstrated that the differences in flexural rigidity and diameters from both tests could be significant. This makes it very difficult to compare these results to earlier results obtained by other researchers, as the stage of plant growth was often omitted.

\section{Acknowledgements}

This work was supported by the National Science Centre, Poland, grant No. UMO-2014/13/D/ST10/01123: "Field experimental investigation of hydrodynamics of water flow-vegetation-sediment interactions at the scale of individual aquatic plants." The authors wish to thank the Ph.D. studies at the Institute of Geophysics of the Polish Academy of Sciences; and Geophysical Observatory of the Institute of Geophysics PAS, the Institute of Meteorology and Water Management, and the Head Office of Geodesy and Cartography for providing the data used in this paper.

\section{Conflict of Interest}

The authors declare no conflict of interest.

\section{References}

1. NEPF H.M. Hydrodynamics of vegetated channels. J. Hydraul. Res. 50 (3), 262, 2012.

2. NIKORA V., LARNED S., NIKORA N., DEBNATH K., COOPER G., REID M. Hydraulic resistance due to aquatic vegetation in small streams: field study. J. Hydraul. Eng. 134 (9), 1326, 2008.

3. ABERLE J., JÄRVELÄ J. Flow resistance of emergent rigid and flexible floodplain vegetation. J. Hydraul. Res. 51 (1), 33, 2013.

4. VÄSTILÄ K., JÄRVELÄ J., KOIVUSALO H. Flowvegetation-sediment interaction in cohesive compound channel. J. Hydraul. Eng. 142 (1), 04015034, 2016. 
5. STEPHAN U., GUTKNECHT D. Hydraulic resistance of submerged flexible vegetation. J. Hydrol. 269, 27, 2002.

6. ROOD S.B., SAMUELSON G.M., BRAATNE J.H., GOURLEY C.R., HUGHES F.M.R., MAHONEY J.M. Managing river flows to restore floodplain forests. Front. Ecol. Environ. 3 (4), 193, 2005.

7. STROMBERG J.C., BEAUCHAMP V.B., DIXON M.D., LITE S.J., PARADZICK C. Importance of low-flow and high-flow characteristics to restoration of riparian vegetation along rivers in arid south-western United States. Freshw. Biol. 52, 651, 2007.

8. JELINEK S., TE T., GEHRIG S.L., STEWART H., NICOL J.M. Facilitating the restoration of aquatic plant communities in a Ramsar wetland. Restor. Ecol. 24 (4), 528, 2016.

9. MOODY M.L., LES D.H., DITOMASO J.M. The role of plant systematic in invasive aquatic plant management. J. Aquat. Plant Manage. 46, 7, 2008.

10. HUSSNER A., STIERS I., VERHOFSTAD M.J.J.M., BAKKER E.S., GRUTTERS B.M.C, HAURY J., VAN VALKENBURG J.L.C.H., BRUNDU G., NEWMAN J., CLAYTON J.S., ANDERSON L.W.J., HOFSTRA D. Management and control methods of invasive alien freshwater aquatic plants: a review. Aquat. Bot. 136, 112, 2017.

11. AYKURT C., FEHRER J., SAN D., KAPLAN Z., DENIZ G., AYDEMIR E., IMIR N. Hybridization between the linear-leaved Potamogeton species in Turkey. Aquat. Bot. 141, 22, 2017.

12. O'HARE M. Aquatic vegetation - a primer for hydrodynamic specialists. J. Hydraul. Res. 53 (6), 687, 2015.

13. BORNETTE G., PUIJALON S. Response of aquatic plants to abiotic factors: a review. Aquat. Sci. 73 (1) 1, 2011.

14. RIIS T., OLESEN B., CLAYTON J.S., LAMBERTINI C., BRIX H., SORRELL B.K. Growth and morphology in relation to temperature and light availability during the establishment of three invasive aquatic plant species. Aquat. Bot. 102, 56, 2012.

15. DE LOS SANTOS C.B., GODBOLD J.A., SOLAN M. Short-term growth and biomechanical responses of the temperate seagrass Cymodocea nodosa to $\mathrm{CO}_{2}$ enrichment. Marine Ecol. Prog. Series 572, 91, 2017.

16. MILER O., ALBAYRAK I., NIKORA V., O'HARE M. Biomechanical properties and morphological characteristics of lake and river plants: implications for adaptations to flow conditions. Aquat. Sci. 76, 465, 2014.

17. KŁOSOWSKI S., KŁOSOWSKI G. Aquatic and marsh plants. Warsaw: MULTICO Oficyna Wyd., 2007 [In Polish].

18. VAN WIJK R.J. Ecological studies on Potamogeton pectinatus LI General characteristics, biomass production and life cycles under field conditions. Aquat. Bot., 31 (3-4), 211, 1988.

19. IIDA S., KOSUGE K., KADONO Y. Molecular phylogeny of Japanese Potamogeton species in light of noncoding chloroplast sequences. Aquat. Bot., 80, 115, 2004.
20. WOOLF T. Chapter 15:3: Curlyleaf Pondweed. In: Biology and control of aquatic plants: a best management practices handbook (Getttys LA, Haller WT, Bellaud M, Eds.). Aquatic Ecosystem Restoration Foundation, Marietta, GA. 125, 2014.

21. MIKULYUK A., NAULR M.E. Curly-leaf Pondweed (Potamogeton crispus): A Technical Review of Distribution, Ecology, Impacts, and Management. Wisconsin Department of Natural Resources Bureau of Science Services, PUB-SS-1052 2009. Madison, Wisconsin, USA, 2009.

22. BREWER C.A., PARKER M. Adaptations of macrophytes to life in moving water: upslope limits and mechanical properties of stems. Hydrobiologia 194, 133, 1990.

23. BOCIĄG K., GAŁKA A., ŁAZARKIEWICZ T., SZMEJA J. Mechanical strength of stems in aquatic macrophytes. Acta Soc. Bot. Pol. 78, 181, 2009.

24. BIALIK R.J., KARPIŃSKI M., RAJWA A., LUKS B., ROWIŃSKI P.M. Bedform characteristics in natural and regulated channels: a comparative field study on the Wilga River, Poland. Acta Geophys. 62 (6), 1413, 2014.

25. ŁOBODA A.M., PRZYBOROWSKI Ł., KARPIŃSKI M., BIALIK R.J., NIKORA V.I. Biomechanical properties of aquatic plants: The effect of test conditions. Limnol. Oceanogr.: Methods 16, 222, 2018.

26. NIKLAS K.J. Plant Biomechanics. An Engineering approach to plant form and function. Univ. Chicago Press, Chicago, 1992.

27. ASTM D790-03. Standard Test Methods for Flexural Properties of Unreinforced and Reinforced Plastics and Electrical Insulating Materials, ASTM International, West Conshohocken, PA, 2003.

28. EDGINGTON E.S. Randomization tests. $3^{\text {rd }}$ ed. MarcelDekker, New York, 1995.

29. RAJWA-KULIGIEWICZ A., BIALIK R.J., ROWIŃSKI P.M. Experimental Investigations on the Oxygen Transfer Efficiency at Low-Head Hydraulics Structures. In Hydrodynamics and Mass Transport at Freshwater Aquatic Interfaces; Rowiński P.M., Marion A., Springer International Publishing: Switzerland, 115, 2016.

30. NIKORA V. Hydrodynamics of aquatic ecosystems: an interface between ecology, biomechanics and environmental fluid mechanics. River Res. Appl. 26 (4), $367,2010$.

31. ROBIONEK A., BANAŚ K., CHMARA R., SZMEJA J. The avoidance strategy of environmental constraints by an aquatic plant Potamogeton alpinus in running waters. Ecol. Evol. 5 (16), 3327, 2015.

32. SCHUTTEN J., DAINTY J., DAVY A.J. Root anchorage and its significance for submerged plants in shallow lakes. J. Ecol. 93 (3), 556, 2005.

33. PUIJALON S., BOUMA T.J., DOUADY C.J., VAN GROENENDAEL J., ANTEN N.P., MARTEL E., BORNETTE G. Plant resistance to mechanical stress: evidence of an avoidance-tolerance trade off. New Phytol. 191 (4), 1141, 2011. 
